\title{
Large deviations for stochastic heat equation with rough dependence in space
}

\author{
YAOZHONG HU ${ }^{1}$, DAVID NUALART ${ }^{1}$ and TUSHENG ZHANG ${ }^{2}$ \\ ${ }^{1}$ Department of Mathematics, University of Kansas, 405 Snow Hall, Lawrence, Kansas, USA. \\ E-mail:yhu@ku.edu; nualart@ku.edu \\ ${ }^{2}$ School of Mathematics, University of Manchester, Oxford Road, Manchester, M13 9PL, UK. \\ E-mail: tusheng.zhang@manchester.ac.uk
}

In this paper, we establish a large deviation principle for the nonlinear one-dimensional stochastic heat equation driven by a Gaussian noise which is white in time and which has the covariance of a fractional Brownian motion with Hurst parameter $H \in\left(\frac{1}{4}, \frac{1}{2}\right)$ in the space variable.

Keywords: fractional Brownian motion; large deviations; stochastic heat equation

\section{Introduction}

In this paper, we consider the one-dimensional stochastic partial differential equations

$$
\frac{\partial u^{\varepsilon}}{\partial t}=\frac{\kappa}{2} \frac{\partial^{2} u^{\varepsilon}}{\partial x^{2}}+\sqrt{\varepsilon} \sigma\left(u^{\varepsilon}\right) \dot{W}, \quad t \in[0, T], x \in \mathbb{R},
$$

where $W$ is a zero-mean Gaussian process with covariance given by

$$
\mathbf{E}[W(s, x) W(t, y)]=\frac{1}{2}\left(|x|^{2 H}+|y|^{2 H}-|x-y|^{2 H}\right) \quad(s \wedge t),
$$

with $\frac{1}{4}<H<\frac{1}{2}$ and $\varepsilon>0$. That is, $W$ is a standard Brownian motion in time and a fractional Brownian motion with Hurst parameter $H$ in the space variable and $\dot{W}=\frac{\partial^{2} W}{\partial t \partial x}$.

The covariance of the noise $\dot{W}$ is given by

$$
\mathbf{E}[\dot{W}(s, x) \dot{W}(t, y)]=\Lambda(x-y) \delta_{0}(t-s),
$$

where $\Lambda$ is a distribution, whose Fourier transform is the measure $\mu(d \xi)=c_{1, H}|\xi|^{1-2 H} d \xi$, with $c_{1, H}$ given in (2.2). Because $\Lambda$ (that can be formally written as $\Lambda(x-y)=H(2 H-1) \times$ $|x-y|^{2 H-2}$ ) is not a locally integrable function, the classical approach developed, among others, by Da Prato and Zabczyk [9], Peszat and Zabczyk [14], Dalang in [7,8] and Dalang and Quer-Sardanyons [6], cannot be applied to such rough covariance. In [1], Balan, Jolis and QuerSardanyons proved the existence and uniqueness of a mild solution to equation (1.1) in the particular case $\sigma(u)=a u+b$, and assuming that the initial condition $u_{0}$ is bounded and Hölder 
continuous of order $H$. The stochastic integral is understood in the Itô sense. The case of a general nonlinear coefficient $\sigma$, which has a Lipschitz derivative and satisfies $\sigma(0)=0$, has been considered by Hu, Huang, Lê, Nualart and Tindel in [13]. In that paper, the existence and uniqueness of a mild solution whose trajectories belong to a suitable space of trajectories is proved by using techniques inspired by the works of Gyöngy [11] and Gyöngy and Nualart [12]. The initial condition $u_{0}$ satisfies some restrictive conditions (see Theorem 2.7).

The purpose of this paper is to establish a large deviation principle for the laws of the solutions $u^{\varepsilon}$ to equation (1.1). For this, we use the weak convergence approach to large deviations based on the Laplace principle, developed by P. Dupuis and R. Ellis [10]. This approach has proved to be successful in a wide range of infinite-dimensional equations (see, for instance, Sritharan and Sundar [16], Chueshov and Millet [5], Chenal and Millet [4], Budhiraja and Dupuis [2], Budhiraja, Dupuis and Maraoulas [3] and Xu and Zhang [17]). In our case, we use the weak convergence of probability measures in the space of trajectories $X_{T}^{\frac{1}{2}-H}$ introduced in Definition 2.6.

The paper is organized as follows. A section of preliminaries contains the definition of stochastic integral, the notion of solution for equation (1.1) and the functional spaces introduced in [13]. In Section 3, we recall a general criteria for large deviations based on weak convergence in [3]. Section 4 is devoted to show the existence and uniqueness of a solution to the skeleton equation associated to equation (1.1) and the stability with respect to perturbations of the skeleton. Finally, in Section 5, we prove the large deviation principle for equation (1.1).

\section{Preliminaries}

Let $\mathcal{D}(\mathbb{R})$ denote the space of real-valued infinitely differentiable functions with compact support on $\mathbb{R}$. The noise $W$ can be represented (see $[13,15])$ as a zero-mean Gaussian family $\left\{W_{t}(\varphi), t \in\right.$ $[0, T], \varphi \in \mathcal{D}(\mathbb{R})\}$ defined on a complete probability space $(\Omega, \mathcal{F}, \mathbf{P})$, whose covariance structure is given by

$$
\mathbf{E}\left[W_{t}(\varphi) W_{s}(\psi)\right]=c_{1, H}(t \wedge s) \int_{\mathbb{R}} \mathcal{F} \varphi(\xi) \overline{\mathcal{F} \psi(\xi)}|\xi|^{1-2 H} d \xi,
$$

where $\mathcal{F} \varphi, \mathcal{F} \psi$ stand for the Fourier transforms of $\varphi, \psi$ and

$$
c_{1, H}=\frac{1}{2 \pi} \Gamma(2 H+1) \sin (\pi H) .
$$

The inner product appearing in (2.1) can be expressed in terms of fractional derivatives. One has

$$
\begin{aligned}
& c_{1, H}(t \wedge s) \int_{\mathbb{R}} \mathcal{F} \varphi(\xi) \overline{\mathcal{F} \psi(\xi)}|\xi|^{1-2 H} d \xi \\
& \quad=c_{H}(t \wedge s) \int_{\mathbb{R}} \int_{\mathbb{R}}(\varphi(x+y)-\varphi(x))(\psi(x+y)-\psi(x))|y|^{2 H-2} d x d y,
\end{aligned}
$$

where $c_{H}$ is an appropriate constant, see [15]. 
Let $\mathcal{H}$ denote the Hilbert space obtained by completing $\mathcal{D}(\mathbb{R})$ under the inner product

$$
\begin{aligned}
\langle\varphi, \psi\rangle_{\mathcal{H}} & :=c_{1, H} \int_{\mathbb{R}} \mathcal{F} \varphi(\xi) \overline{\mathcal{F} \psi(\xi)}|\xi|^{1-2 H} d \xi \\
& =c_{H} \int_{\mathbb{R}} \int_{\mathbb{R}}(\varphi(x+y)-\varphi(x))(\psi(x+y)-\psi(x))|y|^{2 H-2} d x d y .
\end{aligned}
$$

Then the Gaussian family $\left\{W_{t}(\varphi), t \in[0, T], \varphi \in \mathcal{H}\right\}$ can be regarded as an $\mathcal{H}$-cylindrical Brownian motion. Let us now recall the stochastic integral presented in [13].

Definition 2.1. For any $t \geq 0$, let $\mathcal{F}_{t}$ be the $\sigma$-algebra generated by $W$ up to time $t$. An elementary process $g$ is a process given by

$$
g(s, x)=\sum_{i=1}^{n} \sum_{j=1}^{m} X_{i, j} \mathbf{1}_{\left(a_{i}, b_{i}\right]}(s) \mathbf{1}_{\left(h_{j}, l_{j}\right]}(x),
$$

where $n$ and $m$ are finite positive integers, $0 \leq a_{1}<b_{1}<\cdots<a_{n}<b_{n} \leq T, h_{j}<l_{j}$ and $X_{i, j}$ are $\mathcal{F}_{a_{i}}$-measurable random variables for $i=1, \ldots, n$. The integral of such a process with respect to $W$ is defined as

$$
\begin{aligned}
\int_{0}^{T} & \int_{\mathbb{R}} g(s, x) W(d s, d x) \\
= & \sum_{i=1}^{n} \sum_{j=1}^{m} X_{i, j} W\left(\mathbf{1}_{\left(a_{i}, b_{i}\right]} \otimes \mathbf{1}_{\left(h_{j}, l_{j}\right]}\right) \\
= & \sum_{i=1}^{n} \sum_{j=1}^{m} X_{i, j}\left[W\left(b_{i}, l_{j}\right)-W\left(a_{i}, l_{j}\right)-W\left(b_{i}, h_{j}\right)+W\left(a_{i}, h_{j}\right)\right] .
\end{aligned}
$$

One can now extend the notion of integral with respect to $W$ to a broad class of adapted processes.

Proposition 2.2 (1). Let $\Lambda_{H}$ be the space of predictable processes $g$ defined on $[0, T] \times \mathbb{R}$ such that almost surely $g \in L^{2}([0, T] ; \mathcal{H})$ and $\mathbf{E}\left[\int_{0}^{T}\|g(s)\|_{\mathcal{H}}^{2} d s\right]<\infty$. Then, we have:

(i) The space of elementary processes defined in Definition 2.1 is dense in $\Lambda_{H}$.

(ii) For $g \in \Lambda_{H}$, the stochastic integral $\int_{0}^{T} \int_{\mathbb{R}} g(s, x) W(d s, d x)$ is defined as the $L^{2}(\Omega)$-limit of Riemann sums along elementary processes approximating $g$, and we have

$$
\mathbf{E}\left[\left(\int_{0}^{T} \int_{\mathbb{R}} g(s, x) W(d s, d x)\right)^{2}\right]=\mathbf{E}\left[\int_{0}^{T}\|g(s)\|_{\mathcal{H}}^{2} d s\right] .
$$

We recall the definition of the solution to equation (1.1) from [13]. 
Definition 2.3. Suppose $u_{0}$ is a bounded function on $\mathbb{R}$. Let $u^{\varepsilon}=\left\{u^{\varepsilon}(t, x), 0 \leq t \leq T, x \in \mathbb{R}\right\}$ be a real-valued predictable stochastic process such that for all $t \in[0, T]$ and $x \in \mathbb{R}$ the process $\left\{p_{t-s}(x-y) \sigma\left(u^{\varepsilon}(s, y)\right) \mathbf{1}_{[0, t]}(s), 0 \leq s \leq t, y \in \mathbb{R}\right\}$ is an element of $\Lambda_{H}$, where $p_{t}(x)=$ $\frac{1}{\sqrt{2 \pi \kappa t}} e^{-\frac{x^{2}}{2 \kappa t}}$ is the heat kernel on the real line related to $\frac{\kappa}{2} \Delta$. We say that $u^{\varepsilon}$ is a mild solution of (1.1) if for all $t \in[0, T]$ and $x \in \mathbb{R}$ we have

$$
u^{\varepsilon}(t, x)=p_{t} u_{0}(x)+\sqrt{\varepsilon} \int_{0}^{t} \int_{\mathbb{R}} p_{t-s}(x-y) \sigma\left(u^{\varepsilon}(s, y)\right) W(d s, d y) \quad \text { a.s. }
$$

where the stochastic integral is understood in the sense of Proposition 2.2 and

$$
p_{t} u_{0}(x)=\int_{\mathbb{R}} p_{t}(x-y) u_{0}(y) d y
$$

Now let us recall some of the spaces introduced in [13]. Let $(B,\|\cdot\|)$ be a Banach space equipped with the norm $\|\cdot\|$, and let $\beta \in(0,1), \delta \in(0, \infty]$ be fixed numbers. For every function $f: \mathbb{R} \rightarrow B$, we introduce the functions $\mathcal{N}_{\beta}^{B} f, \mathcal{N}_{\beta}^{B,(\delta)} f: \mathbb{R} \rightarrow[0, \infty]$ defined by

$$
\mathcal{N}_{\beta}^{B} f(x)=\left(\int_{\mathbb{R}}\|f(x+h)-f(x)\|^{2}|h|^{-1-2 \beta} d h\right)^{\frac{1}{2}}
$$

and

$$
\mathcal{N}_{\beta}^{B,(\delta)} f(x)=\left(\int_{|h| \leq \delta}\|f(x+h)-f(x)\|^{2}|h|^{-1-2 \beta} d h\right)^{\frac{1}{2}} .
$$

When $B=\mathbb{R}$, we abbreviate the notations $\mathcal{N}_{\beta}^{\mathbb{R}} f$ as $\mathcal{N}_{\beta} f$ and $\mathcal{N}_{\beta}^{\mathbb{R},(\delta)} f$ as $\mathcal{N}_{\beta}^{(\delta)} f$. Notice that for $\delta=\infty$, the above two quantities defined by (2.8) and (2.9) coincide: $\mathcal{N}_{\beta}^{B,(\infty)} f=\mathcal{N}_{\beta}^{B} f$.

Definition 2.4. Let $\mathfrak{X}_{T}^{\beta}(B)$ be the space of all continuous functions $f:[0, T] \times \mathbb{R} \rightarrow B$ such that

$$
\|f\|_{\mathfrak{X}_{T}^{\beta}(B)}:=\sup _{t \in[0, T], x \in \mathbb{R}}\|f(t, x)\|+\sup _{t \in[0, T], x \in \mathbb{R}} \mathcal{N}_{\beta}^{B} f(t, x)<\infty
$$

It was shown in [13] that $\mathfrak{X}_{T}^{\beta}(B)$ is a Banach space. Throughout this paper, we write $\mathfrak{X}_{T}^{p}$ for $\mathfrak{X}_{T}^{\beta}(B)$ with $B=L^{p}(\Omega), \beta=\frac{1}{2}-H$. For $\theta>0$, define the following seminorm for $f$ : $[0, T] \times \mathbb{R} \rightarrow L^{p}(\Omega):$

$$
\|f\|_{\mathfrak{X}_{T, \theta}^{p}}:=\sup _{t \in[0, T], x \in \mathbb{R}} e^{-\theta t}\|f(t, x)\|+\sup _{t \in[0, T], x \in \mathbb{R}} e^{-\theta t} \mathcal{N}_{\frac{1}{2}-H}^{L^{p}(\Omega)} f(t, x) .
$$

For the uniqueness of the solution to (1.1), we need another space. 
Definition 2.5. $\mathcal{Z}_{T}^{p}$ is defined as the space of all random field $f:[0, T] \times \mathbb{R} \times \Omega \rightarrow \mathbb{R}$ such that

$$
\|f\|_{\mathcal{Z}_{T}^{p}}=\sup _{t \in[0, T]}\|f(t, \cdot)\|_{L^{p}(\Omega \times \mathbb{R})}+\sup _{t \in[0, T]} \mathcal{N}_{\frac{1}{2}-H, p}^{*} f(t)<\infty
$$

where $p \geq 2$ and

$$
\mathcal{N}_{\frac{1}{2}-H, p}^{*} f(t)=\left(\int_{\mathbb{R}}\|f(t, \cdot)-f(t, \cdot+h)\|_{L^{p}(\Omega \times \mathbb{R})}^{2}|h|^{2 H-2} d h\right)^{\frac{1}{2}} .
$$

Denote by $C([0, T] \times \mathbb{R})$ the space of all real-valued continuous functions on $[0, T] \times \mathbb{R}$ equipped with the topology of uniform convergence over compact sets. For every $h \in \mathbb{R}$, let $\tau_{h}$ be the translation map in the spatial variable, that is $\tau_{h} f(t, x)=f(t, x-h)$.

Definition 2.6. Let $X_{T}^{\beta}$ be the space of all functions $f \in C([0, T] \times \mathbb{R})$ such that

(i) $(t, x) \mapsto \mathcal{N}_{\beta}^{(1)} f(t, x)$ is finite and continuous on $[0, T] \times \mathbb{R}$.

(ii) $\lim _{h \downarrow 0} \sup _{t \in[0, T], x \in[-R, R]} \mathcal{N}_{\beta}^{(1)}\left(\tau_{h} f-f\right)(t, x)=0$ for every positive $R$.

It turns out that $X_{T}^{\beta}$ is a complete separable metric space equipped with the following topology. A sequence $\left\{f_{n}\right\}$ in $X_{T}^{\beta}$ converges to $f$ in $X_{T}^{\beta}$ if for all $R>0$, the sequences $\left\{f_{n}\right\}$ and $\left\{\mathcal{N}_{\beta}^{(1)}\left(f_{n}-f\right)\right\}$ converge uniformly on $[0, T] \times[-R, R]$ to $f$ and 0 , respectively. We define a metric on $X_{T}^{\beta}$ as follows

$$
d_{\beta}(f, g)=\sum_{n=1}^{\infty} 2^{-n} \frac{\|f-g\|_{n, \beta}}{1+\|f-g\|_{n, \beta}},
$$

where $\|\cdot\|_{n, \beta}$ is the seminorm

$$
\|f\|_{n, \beta}:=\sup _{t \in[0, T], x \in[-n, n]}|f(t, x)|+\sup _{t \in[0, T], x \in[-n, n]} \mathcal{N}_{\beta}^{(1)} f(t, x) .
$$

The following theorem was proved in [13].

Theorem 2.7. Assume that for equation (1.1) the following conditions hold:

(1) The initial condition $u_{0}$ is bounded and locally Hölder continuous of order H. Furthermore, for some $p>\frac{6}{4 H-1}, u_{0}$ is in $L^{p}(\mathbb{R})$ and

$$
\int_{\mathbb{R}}\left\|u_{0}(\cdot)-u_{0}(\cdot+h)\right\|_{L^{p}(\mathbb{R})}^{2}|h|^{2 H-2} d h<\infty .
$$

(2) $\sigma$ is differentiable, its derivative is Lipschitz and $\sigma(0)=0$.

Then there exists a unique solution $u^{\varepsilon}$ to (1.1) in $\mathcal{Z}_{T}^{p} \cap \mathfrak{X}_{T}^{p}$. In addition, the solution has sample paths in the space $X_{T}^{\frac{1}{2}-H}$. 
Condition (2.14) together with $u_{0} \in L^{p}(\mathbb{R})$ for some $p>\frac{6}{4 H-1}$, are required for the uniqueness of the solution in the space $\mathcal{Z}_{T}^{p}$. On the other hand, the boundedness and local Hölder continuity of order $H$ are slightly stronger than the conditions imposed in Theorem 4.25 of [13] for the existence because they imply that $\mathcal{N}_{\beta_{0}} u_{0}$ is bounded for any $\beta_{0}<H$ and we can take $\beta_{0}>\frac{1}{2}-H$. The Hölder continuity of $u_{0}$ will be a useful ingredient in the proof of Theorem 4.3.

Throughout the paper, $C$ will denote a generic constant whose value may change from line to line.

\section{A criteria for large deviations}

Let $\mathcal{H}$ be the Hilbert space introduced in Section 1. Define the following space of stochastic processes:

$$
\mathcal{L}_{2}:=\left\{\psi: \Omega \times[0, T] \rightarrow \mathcal{H} \text { is predictable and } \int_{0}^{T}\|\psi(s)\|_{\mathcal{H}}^{2} d s<\infty \text {, a.s.-P }\right\}
$$

Define $L_{T}(f):=\frac{1}{2} \int_{0}^{T}\|f(s)\|_{\mathcal{H}}^{2} d s$ for $f \in \mathcal{L}_{2}$. Let $\mathbb{U}$ be a Polish space. Set $\mathbb{V}=$ $C([0, T] ; \mathcal{H}) \subseteq C([0, T] \times \mathbb{R})$. Let $\left\{\mathcal{G}^{\varepsilon}\right\}_{\varepsilon>0}$ be a family of measurable maps from $\mathbb{V}$ to $\mathbb{U}$. We present below a sufficient condition for large deviation principle (LDP in abbreviation) to hold for the family $Z^{\varepsilon}=\mathcal{G}^{\varepsilon}(\sqrt{\varepsilon} W)$, as $\varepsilon \rightarrow 0$, where and throughout this section $W$ is the Gaussian process identified as an $\mathcal{H}$-cylindrical Brownian motion.

For $N \geq 1$, define

$$
S^{N}=\left\{f: L^{2}([0, T] ; \mathcal{H}): L_{T}(f) \leq N\right\} .
$$

$S^{N}$ will be equipped with the topology of weak convergence in $L^{2}([0, T] ; \mathcal{H})$.

Set $\mathbb{S}=\bigcup_{N \geq 1} S^{N}$, and

$$
\mathcal{U}^{N}=\left\{f \in \mathcal{L}_{2}: f(\omega) \in S^{N}, \mathbf{P} \text { a.s. } \omega\right\} .
$$

The following condition will be sufficient to establish a LDP for a family $\left\{Z^{\varepsilon}\right\}_{\varepsilon>0}$ defined by $Z^{\varepsilon}=\mathcal{G}^{\varepsilon}(\sqrt{\varepsilon} W)$.

Assumption A. There exists a measurable map $\mathcal{G}^{0}: \mathbb{V} \rightarrow \mathbb{U}$ such that the following hold.

(a) For $N \in \mathbb{N}$, let $f_{n}, f \in S^{N}$ be such that $f_{n} \rightarrow f$ weakly as $n \rightarrow \infty$. Then

$$
\mathcal{G}^{0}\left(\int_{0} f_{n}(s) d s\right) \rightarrow \mathcal{G}^{0}\left(\int_{0}^{\cdot} f(s) d s\right) \quad \text { in } \mathbb{U} .
$$

(b) For $N \in \mathbb{N}$, let $\psi_{\varepsilon}, \psi \in \mathcal{U}^{N}$ be such that $\psi_{\varepsilon}$ converges in distribution to $\psi$ as $\varepsilon \rightarrow 0$. Then

$$
\mathcal{G}^{\varepsilon}\left(W+\frac{1}{\sqrt{\varepsilon}} \int_{0}^{.} \psi_{\varepsilon}(s) d s\right) \Rightarrow \mathcal{G}^{0}\left(\int_{0}^{\cdot} \psi(s) d s\right) \quad \text { in distribution. }
$$


For $\phi \in \mathbb{U}$, define $\mathbb{S}_{\phi}=\left\{f \in \mathbb{S}: \phi=\mathcal{G}^{0}\left(\int_{0}^{\cdot} f(s) d s\right)\right\}$. Let $I: \mathbb{U} \rightarrow[0, \infty]$ be defined by

$$
I(\phi)=\inf _{f \in \mathbb{S}_{\phi}}\left\{L_{T}(f)\right\}, \quad \phi \in \mathbb{U} .
$$

By convention, $I(\phi)=\infty$ if $\mathbb{S}_{\phi}=\varnothing$.

The following criteria was established in [3].

Theorem 3.1. For $\varepsilon>0$, let $Z^{\varepsilon}$ be defined by $Z^{\varepsilon}=\mathcal{G}^{\varepsilon}(\sqrt{\varepsilon} W)$, and suppose that Assumption A holds. Then $I(\phi)$ defined by (3.3) is a rate function on $\mathbb{U}$ and the family $\left\{Z^{\varepsilon}\right\}_{\varepsilon>0}$ satisfies a large deviation principle with rate function $I$.

\section{Skeleton equations}

In this section, we will study the corresponding skeleton equation of the stochastic heat equation (1.1). Let $\left\{e_{k}, k \geq 1\right\}$ be an orthonormal basis of the Hilbert space $\mathcal{H}$. The fractional sheet $W$ admits a representation:

$$
W=\sum_{k=1}^{\infty} \beta_{k}(t) e_{k}
$$

where $\left\{\beta_{k}(t), k \geq 1\right\}$ is a family of independent Brownian motions. The stochastic integral against $W$ can be expressed as

$$
\int_{0}^{T} \int_{\mathbb{R}} g(s, x) W(d s, d x)=\sum_{k=1}^{\infty} \int_{0}^{T}\left\langle g(s, \cdot), e_{k}\right\rangle_{\mathcal{H}} d \beta_{k}(s)
$$

For $f \in \mathbb{S}$, consider the skeleton equation:

$$
u^{f}(t, x)=p_{t} u_{0}(x)+\int_{0}^{t}\left\langle p_{t-s}(x-\cdot) \sigma\left(u^{f}(s, \cdot)\right), f(s, \cdot)\right\rangle_{\mathcal{H}} d s, \quad t \in[0, T], x \in \mathbb{R} .
$$

Before we state the result on the existence and uniqueness of the solution of the above equation, let us first give the following lemma which will be used several times in the rest of the paper.

Lemma 4.1. The following estimates hold:

(1) For any $0 \leq s<t$,

$$
\begin{gathered}
\int_{\mathbb{R}}|z|^{2 H-2} d z \int_{\mathbb{R}^{2}} \mid\left(p_{t-s}\left(z+z_{1}-z_{2}\right)-p_{t-s}\left(z_{1}-z_{2}\right)\right) \\
\quad-\left.\left(p_{t-s}\left(z+z_{1}\right)-p_{t-s}\left(z_{1}\right)\right)\right|^{2}\left|z_{2}\right|^{2 H-2} d z_{1} d z_{2} \\
\leq C(t-s)^{-\frac{3}{2}+2 H} .
\end{gathered}
$$


(2) For any $\beta \in(0,1)$ and $s>0$,

$$
\int_{\mathbb{R}^{2}}\left|p_{s}\left(z+z_{1}\right)-p_{s}\left(z_{1}\right)\right|^{2}|z|^{-1-2 \beta} d z_{1} d z \leq C s^{-\frac{1}{2}-\beta} .
$$

Proof. (4.3) is contained in Lemma 3.1 in [13]. We only prove (4.2). Invoking Plancherel's identity, we have

$$
\begin{aligned}
& \int_{\mathbb{R}^{2}}\left|\left(p_{t-s}\left(z+z_{1}-z_{2}\right)-p_{t-s}\left(z_{1}-z_{2}\right)\right)-\left(p_{t-s}\left(z+z_{1}\right)-p_{t-s}\left(z_{1}\right)\right)\right|^{2}\left|z_{2}\right|^{2 H-2} d z_{1} d z_{2} \\
& =\int_{\mathbb{R}^{2}} e^{-\kappa(t-s) \xi^{2}}\left|e^{i \xi\left(z-z_{2}\right)}-e^{-i \xi z_{2}}-e^{i \xi z}+1\right|^{2}\left|z_{2}\right|^{2 H-2} d \xi d z_{2} \\
& =\int_{\mathbb{R}^{2}} e^{-\kappa(t-s) \xi^{2}}\left|e^{i \xi z}-1\right|^{2}\left|e^{-i \xi z_{2}}-1\right|^{2}\left|z_{2}\right|^{2 H-2} d \xi d z_{2}
\end{aligned}
$$

Consequently,

$$
\begin{aligned}
\int_{\mathbb{R}}|z|^{2 H-2} d z \int_{\mathbb{R}^{2}} \mid\left(p_{t-s}\left(z+z_{1}-z_{2}\right)-p_{t-s}\left(z_{1}-z_{2}\right)\right) \\
\quad-\left.\left(p_{t-s}\left(z+z_{1}\right)-p_{t-s}\left(z_{1}\right)\right)\right|^{2}\left|z_{2}\right|^{2 H-2} d z_{1} d z_{2} \\
\leq \int_{\mathbb{R}} e^{-\kappa(t-s) \xi^{2}} d \xi\left(\int_{\mathbb{R}^{2}}\left|e^{i \xi z}-1\right|^{2}\left|e^{-i \xi z_{2}}-1\right|^{2}\left|z_{2}\right|^{2 H-2}|z|^{2 H-2} d z d z_{2}\right) \\
=\int_{\mathbb{R}} e^{-\kappa(t-s) \xi^{2}} d \xi\left(\int_{\mathbb{R}}\left|e^{i \xi z}-1\right|^{2}|z|^{2 H-2} d z\right)^{2} \\
\leq \int_{\mathbb{R}} e^{-\kappa(t-s) \xi^{2}}|\xi|^{2-4 H} d \xi \leq C(t-s)^{-\frac{3}{2}+2 H} .
\end{aligned}
$$

The next theorem is the existence and uniqueness of the solution of the skeleton equation.

\section{Theorem 4.2. Assume}

(1) the initial condition $u_{0}$ satisfies

$$
\sup _{x \in \mathbb{R}}\left|u_{0}(x)\right|+\sup _{x \in \mathbb{R}} \mathcal{N}_{\frac{1}{2}-H} u_{0}(x)<\infty
$$

(2) $\sigma$ is differentiable, its derivative is Lipschitz and $\sigma(0)=0$.

Then, there exists a unique solution $u^{f}$ to equation (4.1). Moreover, the solution $u^{f}$ belongs to the space $X_{T}^{\frac{1}{2}-H}$. 
Proof. We will solve the equation using a successive iteration in the space $\mathfrak{X}_{T}^{\frac{1}{2}-H}$. Let $\beta:=$ $\frac{1}{2}-H$ in the proof to simplify the presentation. Recall

$$
\|Z\|_{\mathfrak{X}_{T}^{\beta}}=\sup _{0 \leq t \leq T, x \in \mathbb{R}}|Z(t, x)|+\sup _{0 \leq t \leq T, x \in \mathbb{R}}\left|\mathcal{N}_{\beta} Z(t, x)\right| .
$$

We will also use the notation $\|g\|_{\infty}=\sup _{x}|g(x)|$ for a real-valued function $g$ on $\mathbb{R}$.

Define $u_{0}^{f}(t, x)=p_{t} u_{0}(x)$ and

$$
u_{n+1}^{f}(t, x)=p_{t} u_{0}(x)+\int_{0}^{t}\left\langle p_{t-s}(x-\cdot) \sigma\left(u_{n}^{f}(s, \cdot)\right), f(s, \cdot)\right\rangle_{\mathcal{H}} d s .
$$

From the assumptions on $u_{0}$ it follows that $\left\|u_{0}^{f}\right\|_{\mathfrak{X}_{T}^{\beta}}<\infty$. First, we will provide a uniform bound $\left\|u_{n+1}^{f}\right\|_{\mathfrak{X}_{T}^{\beta}}$. From the equation (4.7), we have

$$
\left|u_{n+1}^{f}(t, x)\right|^{2} \leq C\left\|u_{0}\right\|_{\infty}^{2}+C\left(\int_{0}^{T}\|f(s, \cdot)\|_{\mathcal{H}}^{2} d s\right) \int_{0}^{t}\left\|p_{t-s}(x-\cdot) \sigma\left(u_{n}^{f}(s, \cdot)\right)\right\|_{\mathcal{H}}^{2} d s
$$

Moreover,

$$
\begin{aligned}
& \left\|p_{t-s}(x-\cdot) \sigma\left(u_{n}^{f}(s, \cdot)\right)\right\|_{\mathcal{H}}^{2} \\
& =c_{H} \int_{\mathbb{R}^{2}}\left|p_{t-s}(x-(y+z)) \sigma\left(u_{n}^{f}(s, y+z)\right)-p_{t-s}(x-y) \sigma\left(u_{n}^{f}(s, y)\right)\right|^{2}|z|^{2 H-2} d y d z \\
& \leq C \int_{\mathbb{R}^{2}}\left(p_{t-s}(x-(y+z))-p_{t-s}(x-y)\right)^{2}\left|\sigma\left(u_{n}^{f}(s, y+z)\right)\right|^{2}|z|^{2 H-2} d y d z \\
& \quad+C \int_{\mathbb{R}^{2}} p_{t-s}^{2}(x-y)\left|\sigma\left(u_{n}^{f}(s, y+z)\right)-\sigma\left(u_{n}^{f}(s, y)\right)\right|^{2}|z|^{2 H-2} d y d z \\
& \quad:=I_{1}(t, s, x)+I_{2}(t, s, x) .
\end{aligned}
$$

The term $I_{1}(t, s, x)$ can be bounded as

$$
\begin{aligned}
I_{1}(t, s, x) & \leq C\left\|u_{n}^{f}(s, \cdot)\right\|_{\infty}^{2} \int_{\mathbb{R}^{2}}\left(p_{t-s}(x-(y+z))-p_{t-s}(x-y)\right)^{2}|z|^{2 H-2} d y d z \\
& \leq C\left\|u_{n}^{f}(s, \cdot)\right\|_{\infty}^{2}(t-s)^{-1+H},
\end{aligned}
$$

where the Lipschitz property of $\sigma, \sigma(0)=0$, and Lemma 4.1 have been used. For $I_{2}$ we have

$$
\begin{aligned}
I_{2}(t, s, x) & \leq C \int_{\mathbb{R}^{2}} p_{t-s}^{2}(x-y)\left|u_{n}^{f}(s, y+z)-u_{n}^{f}(s, y)\right|^{2}|z|^{2 H-2} d y d z \\
& \leq C \int_{\mathbb{R}} p_{t-s}^{2}(x-y)\left|\mathcal{N}_{\beta} u_{n}^{f}(s, y)\right|^{2} d y \\
& \leq C\left\|\mathcal{N}_{\beta} u_{n}^{f}(s, \cdot)\right\|_{\infty}^{2}(t-s)^{-\frac{1}{2}} .
\end{aligned}
$$


Putting together (4.8)-(4.11), we get

$$
\begin{aligned}
\left\|u_{n+1}^{f}(t, \cdot)\right\|_{\infty}^{2} \leq & C\left\|u_{0}\right\|_{\infty}^{2}+C \int_{0}^{t}\left\|u_{n}^{f}(s, \cdot)\right\|_{\infty}^{2}(t-s)^{-1+H} d s \\
& +C \int_{0}^{t}\left\|\mathcal{N}_{\beta} u_{n}^{f}(s, \cdot)\right\|_{\infty}^{2}(t-s)^{-\frac{1}{2}} d s
\end{aligned}
$$

Next, we want to establish a bound for $\mathcal{N}_{\beta} u_{n+1}^{f}(s, x)$. Set

$$
\Phi(t, x)=\int_{0}^{t}\left\langle p_{t-s}(x-\cdot) \sigma\left(u_{n}^{f}(s, \cdot)\right), f(s, \cdot)\right\rangle_{\mathcal{H}} d s .
$$

We have

$$
\left|\mathcal{N}_{\beta} u_{n+1}^{f}(s, x)\right|^{2} \leq C\left|\mathcal{N}_{\beta}\left(p_{t} u_{0}(x)\right)\right|^{2}+C\left|\mathcal{N}_{\beta} \Phi(t, x)\right|^{2},
$$

where

$$
\begin{aligned}
& \left|\mathcal{N}_{\beta} \Phi(t, x)\right|^{2} \\
& =\int_{\mathbb{R}}|\Phi(t, x+z)-\Phi(t, x)|^{2}|z|^{2 H-2} d z \\
& =\int_{\mathbb{R}} \mid \int_{0}^{t}\left\langle\left(p_{t-s}(x+z-\cdot)-p_{t-s}(x-\cdot)\right) \sigma\left(u_{n}^{f}(s, \cdot)\right),\left.\left.f(s, \cdot)\right|_{\mathcal{H}} d s\right|^{2}|z|^{2 H-2} d z\right. \\
& \leq C \int_{0}^{T}\|f(s, \cdot)\|_{\mathcal{H}}^{2} d s \\
& \quad \times \int_{\mathbb{R}} \int_{0}^{t}\left\|\left(p_{t-s}(x+z-\cdot)-p_{t-s}(x-\cdot)\right) \sigma\left(u_{n}^{f}(s, \cdot)\right)\right\|_{\mathcal{H}}^{2}|z|^{2 H-2} d s d z \\
& \leq C \int_{\mathbb{R}} \int_{0}^{t}\left\|\left(p_{t-s}(x+z-\cdot)-p_{t-s}(x-\cdot)\right) \sigma\left(u_{n}^{f}(s, \cdot)\right)\right\|_{\mathcal{H}}^{2}|z|^{2 H-2} d s d z .
\end{aligned}
$$

The integrand in the above integral can be estimated as follows

$$
\begin{aligned}
\left\|\left(p_{t-s}(x+z-\cdot)-p_{t-s}(x-\cdot)\right) \sigma\left(u_{n}^{f}(s, \cdot)\right)\right\|_{\mathcal{H}}^{2} \\
=c_{H} \int_{\mathbb{R}^{2}}\left[\left(p_{t-s}\left(x+z-\left(z_{1}+z_{2}\right)\right)-p_{t-s}\left(x-\left(z_{1}+z_{2}\right)\right)\right) \sigma\left(u_{n}^{f}\left(s, z_{1}+z_{2}\right)\right)\right. \\
\left.\quad-\left(p_{t-s}\left(x+z-z_{1}\right)-p_{t-s}\left(x-z_{1}\right)\right) \sigma\left(u_{n}^{f}\left(s, z_{1}\right)\right)\right]^{2}\left|z_{2}\right|^{2 H-2} d z_{1} d z_{2} \\
\leq C \int_{\mathbb{R}^{2}} \mid\left(p_{t-s}\left(x+z-\left(z_{1}+z_{2}\right)\right)-p_{t-s}\left(x-\left(z_{1}+z_{2}\right)\right)\right) \\
\quad-\left.\left(p_{t-s}\left(x+z-z_{1}\right)-p_{t-s}\left(x-z_{1}\right)\right)\right|^{2}\left|\sigma\left(u_{n}^{f}\left(s, z_{1}+z_{2}\right)\right)\right|^{2}\left|z_{2}\right|^{2 H-2} d z_{1} d z_{2}
\end{aligned}
$$




$$
\begin{aligned}
& \quad+C \int_{\mathbb{R}^{2}}\left|p_{t-s}\left(x+z-z_{1}\right)-p_{t-s}\left(x-z_{1}\right)\right|^{2} \\
& \quad \times\left|u_{n}^{f}\left(s, z_{1}+z_{2}\right)-u_{n}^{f}\left(s, z_{1}\right)\right|^{2}\left|z_{2}\right|^{2 H-2} d z_{1} d z_{2} \\
& :=J_{1}(t, s, x, z)+J_{2}(t, s, x, z) .
\end{aligned}
$$

Observe that

$$
\begin{aligned}
J_{1}(t, s, x, z) & \\
\leq & C\left\|u_{n}^{f}(s, \cdot)\right\|_{\infty}^{2} \int_{\mathbb{R}^{2}} \mid\left(p_{t-s}\left(x+z-\left(z_{1}+z_{2}\right)\right)-p_{t-s}\left(x-\left(z_{1}+z_{2}\right)\right)\right) \\
& \quad-\left.\left(p_{t-s}\left(x+z-z_{1}\right)-p_{t-s}\left(x-z_{1}\right)\right)\right|^{2}\left|z_{2}\right|^{2 H-2} d z_{1} d z_{2} \\
= & C\left\|u_{n}^{f}(s, \cdot)\right\|_{\infty}^{2} \int_{\mathbb{R}^{2}} \mid\left(p_{t-s}\left(z+z_{1}-z_{2}\right)-p_{t-s}\left(z_{1}-z_{2}\right)\right) \\
& \quad-\left.\left(p_{t-s}\left(z+z_{1}\right)-p_{t-s}\left(z_{1}\right)\right)\right|^{2}\left|z_{2}\right|^{2 H-2} d z_{1} d z_{2} .
\end{aligned}
$$

Consequently, by Lemma 4.1 we have

$$
\int_{\mathbb{R}} J_{1}(t, s, x, z)|z|^{2 H-2} d z \leq C\left\|u_{n}^{f}(s, \cdot)\right\|_{\infty}^{2}(t-s)^{-\frac{3}{2}+2 H} .
$$

For $J_{2}$, we have

$$
\begin{aligned}
\int_{\mathbb{R}} & J_{2}(t, s, x, z)|z|^{2 H-2} d z \\
\leq & C \int_{\mathbb{R}}|z|^{2 H-2} d z \int_{\mathbb{R}^{2}}\left|p_{t-s}\left(x+z-z_{1}\right)-p_{t-s}\left(x-z_{1}\right)\right|^{2} \\
& \times\left|u_{n}^{f}\left(s, z_{1}+z_{2}\right)-u_{n}^{f}\left(s, z_{1}\right)\right|^{2}\left|z_{2}\right|^{2 H-2} d z_{1} d z_{2} \\
\leq & C\left\|\mathcal{N}_{\beta} u_{n}^{f}(s, \cdot)\right\|_{\infty}^{2} \int_{\mathbb{R}^{2}}\left|p_{t-s}\left(x+z-z_{1}\right)-p_{t-s}\left(x-z_{1}\right)\right|^{2}|z|^{2 H-2} d z d z_{1} \\
\leq & C\left\|\mathcal{N}_{\beta} u_{n}^{f}(s, \cdot)\right\|_{\infty}^{2}(t-s)^{-1+H},
\end{aligned}
$$

where Lemma 4.1 has been used. Combining (4.13)-(4.18) together, we obtain

$$
\begin{aligned}
& \left\|\mathcal{N}_{\beta} u_{n+1}^{f}(t, \cdot)\right\|_{\infty}^{2} \\
& \leq C+C \int_{0}^{t}\left\|u_{n}^{f}(s, \cdot)\right\|_{\infty}^{2}(t-s)^{-\frac{3}{2}+2 H} d s \\
& \quad+C \int_{0}^{t}\left\|\mathcal{N}_{\beta} u_{n}^{f}(s, \cdot)\right\|_{\infty}^{2}(t-s)^{-1+H} d s .
\end{aligned}
$$




\section{Define}

$$
A_{n}(t):=\left\|u_{n}^{f}(t, \cdot)\right\|_{\infty}^{2}+\left\|\mathcal{N}_{\beta} u_{n}^{f}(t, \cdot)\right\|_{\infty}^{2} .
$$

Estimates (4.12), (4.19), together with the condition $H \in\left(\frac{1}{4}, \frac{1}{2}\right)$, imply that we can find $\beta_{0}<1$ such that

$$
A_{n+1}(t) \leq C+C \int_{0}^{t}(t-s)^{-\beta_{0}} A_{n}(s) d s .
$$

By Lemma 4.26 in [13], we conclude that

$$
\sup _{n} \sup _{0 \leq t \leq T} A_{n}(t)=\sup _{n} \sup _{0 \leq t \leq T}\left\{\left\|u_{n}^{f}(t, \cdot)\right\|_{\infty}^{2}+\left\|\mathcal{N}_{\beta} u_{n}^{f}(t, \cdot)\right\|_{\infty}^{2}\right\}<\infty .
$$

Next, we are going to show that $\left\{u_{n}^{f}, n \geq 0\right\}$ constitutes a Cauchy sequence in the space $\mathfrak{X}_{T}^{\frac{1}{2}-H}$. To this end, we bound $\left\|u_{n+1}^{f}(t, \cdot)-u_{n}^{f}(t, \cdot)\right\|_{\infty}^{2}$ and $\left\|\mathcal{N}_{\beta}\left(u_{n+1}^{f}(t, \cdot)-u_{n}^{f}(t, \cdot)\right)\right\|_{\infty}^{2}$ separately. Recall

$$
\begin{aligned}
& u_{n+1}^{f}(t, x)-u_{n}^{f}(t, x) \\
& \quad=\int_{0}^{t}\left\langle p_{t-s}(x-\cdot) \sigma\left(u_{n}^{f}(s, \cdot)\right)-p_{t-s}(x-\cdot) \sigma\left(u_{n-1}^{f}(s, \cdot)\right), f(s, \cdot)\right\rangle_{\mathcal{H}} d s .
\end{aligned}
$$

Hence,

$$
\begin{aligned}
& \left|u_{n+1}^{f}(t, x)-u_{n}^{f}(t, x)\right|^{2} \\
& \quad \leq \int_{0}^{T}\|f(s, \cdot)\|_{\mathcal{H}}^{2} d s \int_{0}^{t}\left\|p_{t-s}(x-\cdot)\left(\sigma\left(u_{n}^{f}(s, \cdot)\right)-\sigma\left(u_{n-1}^{f}(s, \cdot)\right)\right)\right\|_{\mathcal{H}}^{2} d s .
\end{aligned}
$$

Now,

$$
\begin{aligned}
\left\|p_{t-s}(x-\cdot)\left(\sigma\left(u_{n}^{f}(s, \cdot)\right)-\sigma\left(u_{n-1}^{f}(s, \cdot)\right)\right)\right\|_{\mathcal{H}}^{2} \\
=c_{H} \int_{\mathbb{R}^{2}} \mid p_{t-s}(x-(y+z))\left(\sigma\left(u_{n}^{f}(s, y+z)\right)-\sigma\left(u_{n-1}^{f}(s, y+z)\right)\right) \\
\quad-\left.p_{t-s}(x-y)\left(\sigma\left(u_{n}^{f}(s, y)\right)-\sigma\left(u_{n-1}^{f}(s, y)\right)\right)\right|^{2}|z|^{2 H-2} d y d z \\
\leq C \int_{\mathbb{R}^{2}}\left(p_{t-s}(x-(y+z))-p_{t-s}(x-y)\right)^{2} \\
\quad \times\left(\sigma\left(u_{n}^{f}(s, y+z)\right)-\sigma\left(u_{n-1}^{f}(s, y+z)\right)\right)^{2}|z|^{2 H-2} d y d z \\
\quad+C \int_{\mathbb{R}^{2}} p_{t-s}^{2}(x-y)\left[\left(\sigma\left(u_{n}^{f}(s, y+z)\right)-\sigma\left(u_{n-1}^{f}(s, y+z)\right)\right)\right. \\
\left.\quad-\left(\sigma\left(u_{n}^{f}(s, y)\right)-\sigma\left(u_{n-1}^{f}(s, y)\right)\right)\right]^{2}|z|^{2 H-2} d y d z \\
:=K_{1}(t, s, x)+K_{2}(t, s, x) .
\end{aligned}
$$


Invoking the Lipschitz continuity of $\sigma$ and (2) in Lemma 4.1, we have

$$
\begin{aligned}
& K_{1}(t, s, x) \\
& \qquad C\left\|u_{n}^{f}(s, \cdot)-u_{n-1}^{f}(s, \cdot)\right\|_{\infty}^{2} \\
& \quad \times \int_{\mathbb{R}^{2}}\left(p_{t-s}(x-(y+z))-p_{t-s}(x-y)\right)^{2}|z|^{2 H-2} d y d z \\
& \leq C\left\|u_{n}^{f}(s, \cdot)-u_{n-1}^{f}(s, \cdot)\right\|_{\infty}^{2}(t-s)^{-1+H} .
\end{aligned}
$$

From the inequality (see [13])

$$
\begin{aligned}
& |(\sigma(a)-\sigma(b))-(\sigma(c)-\sigma(d))| \\
& \quad \leq C|(a-b)-(c-d)|+C|a-b|(|a-c|+|b-d|),
\end{aligned}
$$

we have

$$
\begin{aligned}
& K_{2}(t, s, x) \\
& \leq C \int_{\mathbb{R}^{2}} p_{t-s}^{2}(x-y) \mid\left(u_{n}^{f}(s, y+z)-u_{n-1}^{f}(s, y+z)\right) \\
& -\left.\left(u_{n}^{f}(s, y)-u_{n-1}^{f}(s, y)\right)\right|^{2}|z|^{2 H-2} d y d z \\
& +C \int_{\mathbb{R}^{2}} p_{t-s}^{2}(x-y)\left|u_{n}^{f}(s, y+z)-u_{n-1}^{f}(s, y+z)\right|^{2} \\
& \times\left|u_{n}^{f}(s, y+z)-u_{n}^{f}(s, y)\right|^{2}|z|^{2 H-2} d y d z \\
& +C \int_{\mathbb{R}^{2}} p_{t-s}^{2}(x-y)\left|u_{n}^{f}(s, y+z)-u_{n-1}^{f}(s, y+z)\right|^{2} \\
& \times\left|u_{n-1}^{f}(s, y+z)-u_{n-1}^{f}(s, y)\right|^{2}|z|^{2 H-2} d y d z \\
& \leq C\left\|\mathcal{N}_{\beta}\left(u_{n}^{f}(s, \cdot)-u_{n-1}^{f}(s, \cdot)\right)\right\|_{\infty}^{2} \int_{\mathbb{R}} p_{t-s}^{2}(x-y) d y \\
& +C\left\|u_{n}^{f}(s, \cdot)-u_{n-1}^{f}(s, \cdot)\right\|_{\infty}^{2}\left\|\mathcal{N}_{\beta}\left(u_{n}^{f}(s, \cdot)\right)\right\|_{\infty}^{2} \int_{\mathbb{R}} p_{t-s}^{2}(x-y) d y \\
& +C\left\|u_{n}^{f}(s, \cdot)-u_{n-1}^{f}(s, \cdot)\right\|_{\infty}^{2}\left\|\mathcal{N}_{\beta}\left(u_{n-1}^{f}(s, \cdot)\right)\right\|_{\infty}^{2} \int_{\mathbb{R}} p_{t-s}^{2}(x-y) d y \\
& \leq C(t-s)^{-\frac{1}{2}}\left[\left\|\mathcal{N}_{\beta}\left(u_{n}^{f}(s, \cdot)-u_{n-1}^{f}(s, \cdot)\right)\right\|_{\infty}^{2}+\left\|u_{n}^{f}(s, \cdot)-u_{n-1}^{f}(s, \cdot)\right\|_{\infty}^{2}\right],
\end{aligned}
$$

where we have used the fact (see (4.20))

$$
\sup _{n} \sup _{0 \leq s \leq T}\left\|\mathcal{N}_{\beta}\left(u_{n}^{f}(s, \cdot)\right)\right\|_{\infty}^{2}<\infty .
$$


Putting (4.22)-(4.26) together we get

$$
\begin{aligned}
& \left\|u_{n+1}^{f}(t, \cdot)-u_{n}^{f}(t, \cdot)\right\|_{\infty}^{2} \\
& \quad \leq C \int_{0}^{t}(t-s)^{-\frac{1}{2}}\left[\left\|\mathcal{N}_{\beta}\left(u_{n}^{f}(s, \cdot)-u_{n-1}^{f}(s, \cdot)\right)\right\|_{\infty}^{2}\right. \\
& \left.\quad+\left\|u_{n}^{f}(s, \cdot)-u_{n-1}^{f}(s, \cdot)\right\|_{\infty}^{2}\right] d s .
\end{aligned}
$$

By definition,

$$
\begin{aligned}
\mathcal{N}_{\beta}\left(u_{n+1}^{f}(t, x)-u_{n}^{f}(t, x)\right)^{2} & \int_{\mathbb{R}} \mid \int_{0}^{t}\left(\left\langle p_{t-s}(x+z-\cdot)\left(\sigma\left(u_{n}^{f}(s, \cdot)\right)-\sigma\left(u_{n-1}^{f}(s, \cdot)\right)\right), f(s, \cdot)\right\rangle_{\mathcal{H}}\right. \\
& \left.\quad-\left\langle p_{t-s}(x-\cdot)\left(\sigma\left(u_{n}^{f}(s, \cdot)\right)-\sigma\left(u_{n-1}^{f}(s, \cdot)\right)\right), f(s, \cdot)\right\rangle_{\mathcal{H}}\right)\left.d s\right|^{2}|z|^{2 H-2} d z \\
\leq & \left(\int_{0}^{T}\|f(s, \cdot)\|_{\mathcal{H}}^{2} d s\right) \int_{0}^{t} d s \int_{\mathbb{R}} \|\left(p_{t-s}(x+z-\cdot)-p_{t-s}(x-\cdot)\right) \\
& \times\left(\sigma\left(u_{n}^{f}(s, \cdot)\right)-\sigma\left(u_{n-1}^{f}(s, \cdot)\right)\right) \|_{\mathcal{H}}^{2}|z|^{2 H-2} d z .
\end{aligned}
$$

Furthermore,

$$
\begin{aligned}
&\left\|\left(p_{t-s}(x+z-\cdot)-p_{t-s}(x-\cdot)\right)\left(\sigma\left(u_{n}^{f}(s, \cdot)\right)-\sigma\left(u_{n-1}^{f}(s, \cdot)\right)\right)\right\|_{\mathcal{H}}^{2} \\
&=c_{H} \int_{\mathbb{R}^{2}} \mid\left(p_{t-s}\left(x+z-\left(z_{1}+z_{2}\right)\right)-p_{t-s}\left(x-\left(z_{1}+z_{2}\right)\right)\right) \\
& \quad \times\left(\sigma\left(u_{n}^{f}\left(s, z_{1}+z_{2}\right)\right)-\sigma\left(u_{n-1}^{f}\left(s, z_{1}+z_{2}\right)\right)\right) \\
&-\left.\left(p_{t-s}\left(x+z-z_{1}\right)-p_{t-s}\left(x-z_{1}\right)\right)\left(\sigma\left(u_{n}^{f}\left(s, z_{1}\right)\right)-\sigma\left(u_{n-1}^{f}\left(s, z_{1}\right)\right)\right)\right|^{2}\left|z_{2}\right|^{2 H-2} d z_{1} d z_{2} \\
& \leq C \int_{\mathbb{R}^{2}} \mid\left(p_{t-s}\left(x+z-\left(z_{1}+z_{2}\right)\right)-p_{t-s}\left(x-\left(z_{1}+z_{2}\right)\right)\right) \\
&-\left.\left(p_{t-s}\left(x+z-z_{1}\right)-p_{t-s}\left(x-z_{1}\right)\right)\right|^{2} \\
& \times\left|\left(\sigma\left(u_{n}^{f}\left(s, z_{1}+z_{2}\right)\right)-\sigma\left(u_{n-1}^{f}\left(s, z_{1}+z_{2}\right)\right)\right)\right|^{2}\left|z_{2}\right|^{2 H-2} d z_{1} d z_{2} \\
&+C \int_{\mathbb{R}^{2}}\left|p_{t-s}\left(x+z-z_{1}\right)-p_{t-s}\left(x-z_{1}\right)\right|^{2} \\
& \times\left[\left(\sigma\left(u_{n}^{f}\left(s, z_{1}+z_{2}\right)\right)-\sigma\left(u_{n-1}^{f}\left(s, z_{1}+z_{2}\right)\right)\right)-\left(\sigma\left(u_{n}^{f}\left(s, z_{1}\right)\right)\right.\right. \\
&\left.\left.-\sigma\left(u_{n-1}^{f}\left(s, z_{1}\right)\right)\right)\right]^{2}\left|z_{2}\right|^{2 H-2} d z_{1} d z_{2} \\
&:= M_{1}(t, s, x, z)+M_{2}(t, s, x, z) .
\end{aligned}
$$


By a change of variable and Lemma 4.1, we have

$$
\begin{aligned}
\int_{\mathbb{R}} & M_{1}(t, s, x, z)|z|^{2 H-2} d z \\
\leq & C\left\|u_{n}^{f}(s, \cdot)-u_{n-1}^{f}(s, \cdot)\right\|_{\infty}^{2} \int_{\mathbb{R}}|z|^{2 H-2} d z \\
\quad & \times \int_{\mathbb{R}^{2}} \mid\left(p_{t-s}\left(x+z-\left(z_{1}+z_{2}\right)\right)-p_{t-s}\left(x-\left(z_{1}+z_{2}\right)\right)\right) \\
& -\left.\left(p_{t-s}\left(x+z-z_{1}\right)-p_{t-s}\left(x-z_{1}\right)\right)\right|^{2}\left|z_{2}\right|^{2 H-2} d z_{1} d z_{2} \\
\leq & C\left\|u_{n}^{f}(s, \cdot)-u_{n-1}^{f}(s, \cdot)\right\|_{\infty}^{2}(t-s)^{-\frac{3}{2}+2 H} .
\end{aligned}
$$

Invoking the inequality (4.25), we have

$$
\begin{aligned}
M_{2}( & t, s, x, z) \\
\leq & C \int_{\mathbb{R}^{2}}\left|p_{t-s}\left(x+z-z_{1}\right)-p_{t-s}\left(x-z_{1}\right)\right|^{2} \\
& \times\left[\left(u_{n}^{f}\left(s, z_{1}+z_{2}\right)-u_{n-1}^{f}\left(s, z_{1}+z_{2}\right)\right)-\left(u_{n}^{f}\left(s, z_{1}\right)-u_{n-1}^{f}\left(s, z_{1}\right)\right)\right]^{2}\left|z_{2}\right|^{2 H-2} d z_{1} d z_{2} \\
& +C \int_{\mathbb{R}^{2}}\left|p_{t-s}\left(x+z-z_{1}\right)-p_{t-s}\left(x-z_{1}\right)\right|^{2} \\
& \times\left[\left|u_{n}^{f}\left(s, z_{1}+z_{2}\right)-u_{n-1}^{f}\left(s, z_{1}+z_{2}\right)\right|^{2}\right. \\
& \left.\times\left|u_{n}^{f}\left(s, z_{1}+z_{2}\right)-u_{n}^{f}\left(s, z_{1}\right)\right|^{2}\right]\left|z_{2}\right|^{2 H-2} d z_{1} d z_{2} \\
& +C \int_{\mathbb{R}^{2}}\left|p_{t-s}\left(x+z-z_{1}\right)-p_{t-s}\left(x-z_{1}\right)\right|^{2} \\
& \times\left[\left|u_{n}^{f}\left(s, z_{1}+z_{2}\right)-u_{n-1}^{f}\left(s, z_{1}+z_{2}\right)\right|^{2}\right. \\
& \left.\times\left|u_{n-1}^{f}\left(s, z_{1}+z_{2}\right)-u_{n-1}^{f}\left(s, z_{1}\right)\right|^{2}\right]\left|z_{2}\right|^{2 H-2} d z_{1} d z_{2} \\
:= & M_{21}(t, s, x, z)+M_{22}(t, s, x, z)+M_{23}(t, s, x, z) .
\end{aligned}
$$

Integrating against $|z|^{2 H-2} d z$ and using Lemma 4.1 we have

$$
\begin{aligned}
\int_{\mathbb{R}} & M_{21}(t, s, x, z)|z|^{2 H-2} d z \\
\leq & C\left\|\mathcal{N}_{\beta}\left(u_{n}^{f}(s, \cdot)-u_{n-1}^{f}(s, \cdot)\right)\right\|_{\infty}^{2} \\
\quad & \times \int_{\mathbb{R}^{2}}\left|p_{t-s}\left(x+z-z_{1}\right)-p_{t-s}\left(x-z_{1}\right)\right|^{2}|z|^{2 H-2} d z d z_{1} \\
\leq & C(t-s)^{-1+H}\left\|\mathcal{N}_{\beta}\left(u_{n}^{f}(s, \cdot)-u_{n-1}^{f}(s, \cdot)\right)\right\|_{\infty}^{2},
\end{aligned}
$$


and

$$
\begin{aligned}
\int_{\mathbb{R}} & M_{22}(t, s, x, z)|z|^{2 H-2} d z \\
\leq & C\left\|u_{n}^{f}(s, \cdot)-u_{n-1}^{f}(s, \cdot)\right\|_{\infty}^{2} \int_{\mathbb{R}}|z|^{2 H-2} d z \int_{\mathbb{R}^{2}}\left|p_{t-s}\left(x+z-z_{1}\right)-p_{t-s}\left(x-z_{1}\right)\right|^{2} \\
& \times\left|u_{n}^{f}\left(s, z_{1}+z_{2}\right)-u_{n}^{f}\left(s, z_{1}\right)\right|^{2}\left|z_{2}\right|^{2 H-2} d z_{1} d z_{2} \\
\leq & C\left\|u_{n}^{f}(s, \cdot)-u_{n-1}^{f}(s, \cdot)\right\|_{\infty}^{2}\left\|\mathcal{N}_{\beta}\left(u_{n}^{f}(s, \cdot)\right)\right\|_{\infty}^{2} \\
& \times \int_{\mathbb{R}^{2}}\left|p_{t-s}\left(x+z-z_{1}\right)-p_{t-s}\left(x-z_{1}\right)\right|^{2}|z|^{2 H-2} d z d z_{1} \\
\leq & C\left\|u_{n}^{f}(s, \cdot)-u_{n-1}^{f}(s, \cdot)\right\|_{\infty}^{2}\left\|\mathcal{N}_{\beta}\left(u_{n}^{f}(s, \cdot)\right)\right\|_{\infty}^{2}(t-s)^{-1+H} \\
\leq & C\left\|u_{n}^{f}(s, \cdot)-u_{n-1}^{f}(s, \cdot)\right\|_{\infty}^{2}(t-s)^{-1+H} .
\end{aligned}
$$

Notice that (4.20) was used in the last step. Similarly, the following holds true:

$$
\int_{\mathbb{R}} M_{23}(t, s, x, z)|z|^{2 H-2} d z \leq C\left\|u_{n}^{f}(s, \cdot)-u_{n-1}^{f}(s, \cdot)\right\|_{\infty}^{2}(t-s)^{-1+H} .
$$

Then, (4.32), (4.33) and (4.34) together gives

$$
\begin{aligned}
\int_{\mathbb{R}} M_{2}(t, s, x, z)|z|^{2 H-2} d z \leq & C\left\|u_{n}^{f}(s, \cdot)-u_{n-1}^{f}(s, \cdot)\right\|_{\infty}^{2}(t-s)^{-1+H} \\
& +C(t-s)^{-1+H}\left\|\mathcal{N}_{\beta}\left(u_{n}^{f}(s, \cdot)-u_{n-1}^{f}(s, \cdot)\right)\right\|_{\infty}^{2} .
\end{aligned}
$$

Substitute (4.29), (4.30), (4.35) back into (4.28) to obtain

$$
\begin{aligned}
& \left\|\mathcal{N}_{\beta}\left(u_{n+1}^{f}(t, \cdot)-u_{n}^{f}(t, \cdot)\right)\right\|_{\infty}^{2} \\
& \leq C \int_{0}^{t}\left\|u_{n}^{f}(s, \cdot)-u_{n-1}^{f}(s, \cdot)\right\|_{\infty}^{2}(t-s)^{-1+H} d s \\
& \quad+C \int_{0}^{t}(t-s)^{-1+H}\left\|\mathcal{N}_{\beta}\left(u_{n}^{f}(s, \cdot)-u_{n-1}^{f}(s, \cdot)\right)\right\|_{\infty}^{2} d s \\
& \quad+C \int_{0}^{t}\left\|u_{n}^{f}(s, \cdot)-u_{n-1}^{f}(s, \cdot)\right\|_{\infty}^{2}(t-s)^{-\frac{3}{2}+2 H} d s .
\end{aligned}
$$

Setting

$$
R_{n}(t):=\left\|u_{n+1}^{f}(t, \cdot)-u_{n}^{f}(t, \cdot)\right\|_{\infty}^{2}+\left\|\mathcal{N}_{\beta}\left(u_{n+1}^{f}(t, \cdot)-u_{n}^{f}(t, \cdot)\right)\right\|_{\infty}^{2},
$$

it follows from (4.27) and (4.36) that there exists a $\beta_{0}<1$ such that

$$
R_{n+1}(t) \leq C \int_{0}^{t}(t-s)^{-\beta_{0}} R_{n}(s) d s .
$$


Applying Lemma 4.26 in [13], we conclude from (4.37) that $\sum_{n=0}^{\infty} R_{n}^{\frac{1}{p}}$ converges uniformly in $[0, T]$ for all $1 \leq p<\infty$. In particular, this implies that $u_{n}^{f}, n \geq 0$ is a Cauchy sequence in the $\mathfrak{X}_{T}^{\beta}$. Denote by $u^{f}$ the limit of $\left\{u_{n}^{f}\right\}$. Letting $n \rightarrow \infty$ in (4.7), it follows easily that

$$
u^{f}(t, x)=p_{t} u_{0}(x)+\int_{0}^{t}\left\langle p_{t-s}(x-\cdot) \sigma\left(u^{f}(s, \cdot)\right), f(s, \cdot)\right\rangle_{\mathcal{H}} d s .
$$

We have proved the existence of the solution of equation (4.1). The statement, $u^{f} \in X_{T}^{\frac{1}{2}-H}$, follows from the Hölder continuity of $u^{f}$ (see the proof of Theorem 4.3 below) and Lemma 4.12 in [13]. Suppose $u^{f}, v^{f}$ both are solutions to equation (4.1). By the similar estimates as for $u_{n+1}^{f}-u_{n}^{f}$ we can show that

$$
\begin{aligned}
& \left\|u^{f}(t, \cdot)-v^{f}(t, \cdot)\right\|_{\infty}^{2}+\left\|\mathcal{N}_{\beta}\left(u^{f}(t, \cdot)-v^{f}(t, \cdot)\right)\right\|_{\infty}^{2} \\
& \quad \leq C \int_{0}^{t}(t-s)^{-\beta_{0}}\left\{\left\|u^{f}(s, \cdot)-v^{f}(s, \cdot)\right\|_{\infty}^{2}+\left\|\mathcal{N}_{\beta}\left(u^{f}(s, \cdot)-v^{f}(s, \cdot)\right)\right\|_{\infty}^{2}\right\} d s
\end{aligned}
$$

for some $\beta_{0}<1$. This implies

$$
\left\|u^{f}(t, \cdot)-v^{f}(t, \cdot)\right\|_{\infty}^{2}+\left\|\mathcal{N}_{\beta}\left(u^{f}(t, \cdot)-v^{f}(t, \cdot)\right)\right\|_{\infty}^{2}=0
$$

for $t \in[0, T]$, proving the uniqueness.

For $f \in \mathbb{S}$, define a mapping $\mathcal{G}^{0}$ by

$$
\mathcal{G}^{0}\left(\int_{0}^{\cdot} f(s) d s\right)=u^{f}(\cdot)
$$

where $u^{f}$ is the solution to equation (4.1).

Theorem 4.3. Suppose that the following conditions hold:

(1) The initial condition $u_{0}$ is bounded and locally Hölder continuous of order $H$.

(2) $\sigma$ is differentiable, its derivative is Lipschitz and $\sigma(0)=0$.

For $N \in \mathbb{N}$, let $f_{n}, f \in S^{N}$ be such that $f_{n} \rightarrow f$ weakly as $n \rightarrow \infty$. Let $u^{f_{n}}$ denote the solution of equation (4.1) replacing $f$ by $f_{n}$. Then

$$
\mathcal{G}^{0}\left(\int_{0}^{\cdot} f_{n}(s) d s\right)=u^{f_{n}} \rightarrow \mathcal{G}^{0}\left(\int_{0}^{\cdot} f(s) d s\right)=u^{f}
$$

in the space $X_{T}^{\frac{1}{2}-H}$.

Proof. Recall

$$
u^{f_{n}}(t, x)=p_{t} u_{0}(x)+\int_{0}^{t}\left\langle p_{t-s}(x-\cdot) \sigma\left(u^{f_{n}}(s, \cdot)\right), f_{n}(s, \cdot)\right\rangle_{\mathcal{H}} d s .
$$


Since the norm $\left\{\int_{0}^{T}\left\|f_{n}(s)\right\|_{\mathcal{H}}^{2} d s, n \geq 1\right\}$ is bounded by a constant $N$, invoking similar arguments as in the proof of Theorem 4.2, we can show that

$$
\sup _{n} \sup _{0 \leq t \leq T}\left\{\left\|u^{f_{n}}(t, \cdot)\right\|_{\infty}^{2}+\left\|\mathcal{N}_{\frac{1}{2}-H}\left(u^{f_{n}}(t, \cdot)\right)\right\|_{\infty}^{2}\right\}<\infty .
$$

Next, we prove that the family $\left\{u^{f_{n}}, n \geq 1\right\}$ is equi-Hölder continuous. Let

$$
v^{f_{n}}(t, x):=\int_{0}^{t}\left\langle p_{t-s}(x-\cdot) \sigma\left(u^{f_{n}}(s, \cdot)\right), f_{n}(s, \cdot)\right\rangle_{\mathcal{H}} d s .
$$

It is sufficient to show that $\left\{v^{f_{n}}, n \geq 1\right\}$ are Hölder continuous with Hölder constant and Hölder exponent being independent of $n$. For $0 \leq t_{1}<t_{2} \leq T$, we have

$$
\begin{aligned}
& \left|v^{f_{n}}\left(t_{2}, x\right)-v^{f_{n}}\left(t_{1}, x\right)\right|^{2} \\
& \leq C\left|\int_{t_{1}}^{t_{2}}\left\langle p_{t_{2}-s}(x-\cdot) \sigma\left(u^{f_{n}}(s, \cdot)\right), f_{n}(s, \cdot)\right\rangle_{\mathcal{H}} d s\right|^{2} \\
& \quad+C\left|\int_{0}^{t_{1}}\left\langle\left(p_{t_{2}-s}(x-\cdot)-p_{t_{1}-s}(x-\cdot)\right) \sigma\left(u^{f_{n}}(s, \cdot)\right), f_{n}(s, \cdot)\right\rangle_{\mathcal{H}} d s\right|^{2} \\
& \quad C \int_{t_{1}}^{t_{2}}\left\|p_{t_{2}-s}(x-\cdot) \sigma\left(u^{f_{n}}(s, \cdot)\right)\right\|_{\mathcal{H}}^{2} d s \\
& \quad+C \int_{0}^{t_{1}}\left\|\left(p_{t_{2}-s}(x-\cdot)-p_{t_{1}-s}(x-\cdot)\right) \sigma\left(u^{f_{n}}(s, \cdot)\right)\right\|_{\mathcal{H}}^{2} d s .
\end{aligned}
$$

Using the similar arguments as in the proof of (4.9), (4.10) and (4.11), we have

$$
\begin{aligned}
& \left\|p_{t_{2}-s}(x-\cdot) \sigma\left(u^{f_{n}}(s, \cdot)\right)\right\|_{\mathcal{H}}^{2} \\
& \quad \leq C\left\{\left\|u^{f_{n}}(t, \cdot)\right\|_{\infty}^{2}+\left\|\mathcal{N}_{\frac{1}{2}-H}\left(u^{f_{n}}(t, \cdot)\right)\right\|_{\infty}^{2}\right\}\left(t_{2}-s\right)^{-1+H} \\
& \quad \leq C\left(t_{2}-s\right)^{-1+H},
\end{aligned}
$$

where (4.41) has been used. Hence,

$$
\int_{t_{1}}^{t_{2}}\left\|p_{t_{2}-s}(x-\cdot) \sigma\left(u^{f_{n}}(s, \cdot)\right)\right\|_{\mathcal{H}}^{2} d s \leq C \int_{t_{1}}^{t_{2}}\left(t_{2}-s\right)^{-1+H} d s=C\left(t_{2}-t_{1}\right)^{H} .
$$

On the other hand,

$$
\begin{aligned}
& \left\|\left(p_{t_{2}-s}(x-\cdot)-p_{t_{1}-s}(x-\cdot)\right) \sigma\left(u^{f_{n}}(s, \cdot)\right)\right\|_{\mathcal{H}}^{2} \\
& \quad \leq C \int_{\mathbb{R}^{2}}\left[\left(p_{t_{2}-s}(x-(y+z))-p_{t_{1}-s}(x-(y+z))\right)\right.
\end{aligned}
$$




$$
\begin{aligned}
& \left.-\left(p_{t_{2}-s}(x-y)-p_{t_{1}-s}(x-y)\right)\right]^{2}\left|\sigma\left(u^{f_{n}}(s, y+z)\right)\right|^{2}|z|^{2 H-2} d y d z \\
& +C \int_{\mathbb{R}^{2}}\left(p_{t_{2}-s}(x-y)-p_{t_{1}-s}(x-y)\right)^{2} \\
& \times\left|\sigma\left(u^{f_{n}}(s, y+z)\right)-\sigma\left(u^{f_{n}}(s, y)\right)\right|^{2}|z|^{2 H-2} d y d z \\
:= & A_{1}\left(t_{2}, t_{1}, s, x\right)+A_{2}\left(t_{2}, t_{1}, s, x\right) .
\end{aligned}
$$

Applying the Plancherel's identity and (4.41), we have

$$
\begin{aligned}
& A_{1}\left(t_{2}, t_{1}, s, x\right) \\
& \leq C\left\|u^{f_{n}}(s, \cdot)\right\|_{\infty}^{2} \int_{\mathbb{R}^{2}}\left[\left(p_{t_{2}-s}(x-(y+z))-p_{t_{1}-s}(x-(y+z))\right)\right. \\
&\left.-\left(p_{t_{2}-s}(x-y)-p_{t_{1}-s}(x-y)\right)\right]^{2}|z|^{2 H-2} d y d z \\
&= C\left\|u^{f_{n}}(s, \cdot)\right\|_{\infty}^{2} \int_{\mathbb{R}^{2}}\left[\left(p_{t_{2}-s}(y-z)\right)-p_{t_{1}-s}(y-z)\right) \\
&\left.-\left(p_{t_{2}-s}(y)-p_{t_{1}-s}(y)\right)\right]^{2}|z|^{2 H-2} d y d z \\
& \leq C \int_{\mathbb{R}^{2}} \mid\left(e^{-\frac{\kappa}{2}\left(t_{2}-s\right) \xi^{2}} e^{-i \xi z}-e^{-\frac{\kappa}{2}\left(t_{1}-s\right) \xi^{2}} e^{-i \xi z}\right) \\
&-\left.\left(e^{-\frac{\kappa}{2}\left(t_{2}-s\right) \xi^{2}}-e^{-\frac{\kappa}{2}\left(t_{1}-s\right) \xi^{2}}\right)\right|^{2}|z|^{2 H-2} d \xi d z \\
& \leq C \int_{\mathbb{R}^{2}} e^{-\kappa\left(t_{1}-s\right) \xi^{2}}\left|e^{-\frac{\kappa}{2}\left(t_{2}-t_{1}\right) \xi^{2}}-1\right|^{2}\left|e^{-i \xi z}-1\right|^{2}|z|^{2 H-2} d \xi d z .
\end{aligned}
$$

Through a change of variables, it follows that

$$
\begin{aligned}
& \int_{0}^{t_{1}} A_{1}\left(t_{2}, t_{1}, s, x\right) d s \\
& \quad \leq C \int_{\mathbb{R}^{2}} \int_{0}^{t_{1}} e^{-\kappa\left(t_{1}-s\right) \xi^{2}} d s\left|e^{-\frac{\kappa}{2}\left(t_{2}-t_{1}\right) \xi^{2}}-1\right|^{2}\left|e^{-i \xi z}-1\right|^{2}|z|^{2 H-2} d \xi d z \\
& \quad \leq C\left(t_{2}-t_{1}\right)^{H}
\end{aligned}
$$

Similarly, we have

$$
\begin{aligned}
A_{2}\left(t_{2}, t_{1}, s, x\right) & \leq C\left\|\mathcal{N}_{\frac{1}{2}-H}\left(u^{f_{n}}(s, \cdot)\right)\right\|_{\infty}^{2} \int_{\mathbb{R}}\left(p_{t_{2}-s}(y)-p_{t_{1}-s}(y)\right)^{2} d y \\
& \leq C \int_{\mathbb{R}} e^{-\kappa\left(t_{1}-s\right) \xi^{2}}\left|e^{-\frac{\kappa}{2}\left(t_{2}-t_{1}\right) \xi^{2}}-1\right|^{2} d \xi
\end{aligned}
$$


which yields that

$$
\int_{0}^{t_{1}} A_{2}\left(t_{2}, t_{1}, s, x\right) d s \leq C\left(t_{2}-t_{1}\right)^{\frac{1}{2}} .
$$

Putting together (4.42), (4.44), (4.45),(4.47) and (4.49), we see that there exists a constant $C$ (independent of $n$ ) such that

$$
\left|v^{f_{n}}\left(t_{2}, x\right)-v^{f_{n}}\left(t_{1}, x\right)\right|^{2} \leq C\left|t_{2}-t_{1}\right|^{H}, \quad \text { for all } t_{1}, t_{2} \in[0, T], x \in \mathbb{R} .
$$

Let $x_{1}, x_{2} \in \mathbb{R}$ and consider

$$
v^{f_{n}}\left(t, x_{1}\right)-v^{f_{n}}\left(t, x_{2}\right)=\int_{0}^{t}\left\langle\left(p_{t-s}\left(x_{1}-\cdot\right)-p_{t-s}\left(x_{2}-\cdot\right)\right) \sigma\left(u^{f_{n}}(s, \cdot)\right), f_{n}(s, \cdot)\right\rangle_{\mathcal{H}} d s .
$$

We have

$$
\left|v^{f_{n}}\left(t, x_{1}\right)-v^{f_{n}}\left(t, x_{2}\right)\right|^{2} \leq C \int_{0}^{t}\left\|\left(p_{t-s}\left(x_{1}-\cdot\right)-p_{t-s}\left(x_{2}-\cdot\right)\right) \sigma\left(u^{f_{n}}(s, \cdot)\right)\right\|_{\mathcal{H}}^{2} d s .
$$

Now,

$$
\begin{aligned}
\|( & \left.p_{t-s}\left(x_{1}-\cdot\right)-p_{t-s}\left(x_{2}-\cdot\right)\right) \sigma\left(u^{f_{n}}(s, \cdot)\right) \|_{\mathcal{H}}^{2} \\
= & c_{H} \int_{\mathbb{R}^{2}} \mid\left(p_{t-s}\left(x_{1}-(y+z)\right)-p_{t-s}\left(x_{2}-(y+z)\right)\right) \sigma\left(u^{f_{n}}(s, y+z)\right) \\
& -\left.\left(p_{t-s}\left(x_{1}-y\right)-p_{t-s}\left(x_{2}-y\right)\right) \sigma\left(u^{f_{n}}(s, y)\right)\right|^{2}|z|^{2 H-2} d y d z \\
\leq & C \int_{\mathbb{R}^{2}} \mid\left(p_{t-s}\left(x_{1}-(y+z)\right)-p_{t-s}\left(x_{2}-(y+z)\right)\right) \\
& -\left.\left(p_{t-s}\left(x_{1}-y\right)-p_{t-s}\left(x_{2}-y\right)\right)\right|^{2}\left|\sigma\left(u^{f_{n}}(s, y+z)\right)\right|^{2}|z|^{2 H-2} d y d z \\
& +C \int_{\mathbb{R}^{2}}\left|p_{t-s}\left(x_{1}-y\right)-p_{t-s}\left(x_{2}-y\right)\right|^{2}\left|\sigma\left(u^{f_{n}}(s, y+z)\right)-\sigma\left(u^{f_{n}}(s, y)\right)\right|^{2}|z|^{2 H-2} d y d z \\
:= & B_{1}\left(t, s, x_{1}, x_{2}\right)+B_{2}\left(t, s, x_{1}, x_{2}\right) .
\end{aligned}
$$

In view of (4.41) and invoking Plancherel's identity, we have

$$
\begin{aligned}
& B_{1}\left(t, s, x_{1}, x_{2}\right) \\
& \leq C\left\|u^{f_{n}}(s, \cdot)\right\|_{\infty}^{2} \int_{\mathbb{R}^{2}} \mid\left(p_{t-s}\left(x_{1}-(y+z)\right)-p_{t-s}\left(x_{2}-(y+z)\right)\right) \\
& \quad-\left.\left(p_{t-s}\left(x_{1}-y\right)-p_{t-s}\left(x_{2}-y\right)\right)\right|^{2}|z|^{2 H-2} d y d z \\
& \leq C \int_{\mathbb{R}^{2}} e^{-\kappa(t-s) \xi^{2}}\left|e^{-i \xi\left(x_{1}-x_{2}-z\right)}-e^{-i \xi z}-e^{-i \xi\left(x_{1}-x_{2}\right)}+1\right|^{2}|z|^{2 H-2} d \xi d z \\
& =C \int_{\mathbb{R}^{2}} e^{-\kappa(t-s) \xi^{2}}\left|e^{-i \xi\left(x_{1}-x_{2}\right)}-1\right|^{2}\left|e^{-i \xi z}-1\right|^{2}|z|^{2 H-2} d \xi d z .
\end{aligned}
$$


Integrating against $d s$ and using a change of variables, we deduce that

$$
\begin{aligned}
\int_{0}^{t} B_{1}\left(t, s, x_{1}, x_{2}\right) d s & \leq C \int_{\mathbb{R}} \frac{1}{|\xi|^{1+2 H}}\left|e^{-i \xi\left(x_{1}-x_{2}\right)}-1\right|^{2} d \xi \\
& \leq C\left|x_{1}-x_{2}\right|^{2 H}
\end{aligned}
$$

For $B_{2}$, we have

$$
\begin{aligned}
B_{2}\left(t, s, x_{1}, x_{2}\right) & \leq C\left\|\mathcal{N}_{\frac{1}{2}-H}\left(u^{f_{n}}(s, \cdot)\right)\right\|_{\infty}^{2} \int_{\mathbb{R}}\left|p_{t-s}\left(x_{1}-y\right)-p_{t-s}\left(x_{2}-y\right)\right|^{2} d y \\
& \leq C \int_{\mathbb{R}} e^{-\kappa(t-s) \xi^{2}}\left|e^{-i \xi\left(x_{1}-x_{2}\right)}-1\right|^{2} d \xi,
\end{aligned}
$$

which yields that

$$
\begin{aligned}
\int_{0}^{t} B_{2}\left(t, s, x_{1}, x_{2}\right) d s & \leq C \int_{\mathbb{R}} \int_{0}^{t} e^{-\kappa(t-s) \xi^{2}} d s\left|e^{-i \xi\left(x_{1}-x_{2}\right)}-1\right|^{2} d \xi \\
& \leq C \int_{\mathbb{R}} \frac{1}{\xi^{2}}\left|e^{-i \xi\left(x_{1}-x_{2}\right)}-1\right|^{2} d \xi \\
& \leq C\left|x_{1}-x_{2}\right| .
\end{aligned}
$$

Collecting the inequalities in (4.51), (4.52), (4.54) and (4.56) we arrive at

$$
\left|v^{f_{n}}\left(t, x_{1}\right)-v^{f_{n}}\left(t, x_{2}\right)\right|^{2} \leq C\left(\left|x_{1}-x_{2}\right|+\left|x_{1}-x_{2}\right|^{2 H}\right) .
$$

As $u^{f_{n}}(t, x)=p_{t} u_{0}(x)+v^{f_{n}}(t, x)$, it follows from (4.50), (4.57) that there exists an independent constant $C$ such that

$$
\left|u^{f_{n}}\left(t_{1}, x_{1}\right)-u^{f_{n}}\left(t_{2}, x_{2}\right)\right|^{2} \leq C\left\{\left|x_{1}-x_{2}\right|+\left|x_{1}-x_{2}\right|^{2 H}+\left|t_{2}-t_{1}\right|^{H}\right\}
$$

for all $t_{1}, t_{2} \in[0, T], x_{1}, x_{2} \in \mathbb{R}$. The above uniform estimate along with the Arzela-Ascoli theorem yields that there exists a subsequence $\left\{n_{k}, k \geq 1\right\}$ and a uniform continuous function $u(t, x)$ such that

$$
\sup _{0 \leq t \leq T} \sup _{x \in[-R, R]}\left|u^{f_{n_{k}}}(t, x)-u(t, x)\right|^{2} \rightarrow 0
$$

for every $R>0$ as $k \rightarrow \infty$. First, we will show $u=u^{f}$. By the uniqueness of the equation, it is sufficient to show that $u$ is a solution to equation (4.1). Applying Fatou lemma and taking into account (4.41), (4.58) it is easy to see that

$$
\sup _{0 \leq t \leq T}\left\{\|u(t, \cdot)\|_{\infty}^{2}+\left\|\mathcal{N}_{\frac{1}{2}-H}(u(t, \cdot))\right\|_{\infty}^{2}\right\}<\infty
$$

and

$$
\left|u\left(t_{1}, x_{1}\right)-u\left(t_{2}, x_{2}\right)\right|^{2} \leq C\left\{\left|x_{1}-x_{2}\right|+\left|x_{1}-x_{2}\right|^{2 H}+\left|t_{2}-t_{1}\right|^{H}\right\}
$$


for all $t_{1}, t_{2} \in[0, T], x_{1}, x_{2} \in \mathbb{R}$. Recall that

$$
u^{f_{n_{k}}(t, x)}=p_{t} u_{0}(x)+\int_{0}^{t}\left\langle p_{t-s}(x-\cdot) \sigma\left(u^{f_{n_{k}}}(s, \cdot)\right), f_{n_{k}}(s, \cdot)\right\rangle_{\mathcal{H}} d s .
$$

To pass to the limit in the above equation as $k \rightarrow \infty$, we need to prove

$$
\lim _{k \rightarrow \infty} \int_{0}^{t}\left\|p_{t-s}(x-\cdot) \sigma\left(u^{f_{n_{k}}}(s, \cdot)\right)-p_{t-s}(x-\cdot) \sigma(u(s, \cdot))\right\|_{\mathcal{H}}^{2} d s=0 .
$$

In fact,

$$
\begin{aligned}
&\left\|p_{t-s}(x-\cdot)\left(\sigma\left(u^{f_{n_{k}}}(s, \cdot)\right)-\sigma(u(s, \cdot))\right)\right\|_{\mathcal{H}}^{2} \\
& \leq C \int_{\mathbb{R}^{2}}\left(p_{t-s}(x-(y+z))-p_{t-s}(x-y)\right)^{2} \\
& \quad \times\left(\sigma\left(u^{f_{n_{k}}}(s, y+z)\right)-\sigma(u(s, y+z))\right)^{2}|z|^{2 H-2} d y d z \\
&+C \int_{\mathbb{R}^{2}} p_{t-s}^{2}(x-y)\left[\left(\sigma\left(u^{f_{n_{k}}}(s, y+z)\right)-\sigma(u(s, y+z))\right)\right. \\
&\left.\quad-\left(\sigma\left(u^{f_{n_{k}}}(s, y)\right)-\sigma(u(s, y))\right)\right]^{2}|z|^{2 H-2} d y d z \\
&:= D_{1}^{k}(t, s, x)+D_{2}^{k}(t, s, x) .
\end{aligned}
$$

For every $y, z$, clearly $\left(\sigma\left(u^{f_{n_{k}}}(s, y+z)\right)-\sigma(u(s, y+z))\right)^{2} \rightarrow 0$ as $k \rightarrow \infty$. On the other hand,

$$
\begin{aligned}
& \left(p_{t-s}(x-(y+z))-p_{t-s}(x-y)\right)^{2}\left(\sigma\left(u^{f_{n_{k}}}(s, y+z)\right)-\sigma(u(s, y+z))\right)^{2}|z|^{2 H-2} \\
& \quad \leq C\left\|u^{f_{n_{k}}}(s, \cdot)-u(s, \cdot)\right\|_{\infty}^{2}\left(p_{t-s}(x-(y+z))-p_{t-s}(x-y)\right)^{2}|z|^{2 H-2} \\
& \quad \leq C\left(p_{t-s}(x-(y+z))-p_{t-s}(x-y)\right)^{2}|z|^{2 H-2}
\end{aligned}
$$

where the right-hand side is $d y d z$-integrable. Applying the dominated convergence theorem, we deduce that

$$
\lim _{k \rightarrow \infty} D_{1}^{k}(t, s, x)=0
$$

Moreover, by a change of variable and Lemma 4.1 we have

$$
\begin{aligned}
D_{1}^{k}(t, s, x) & \leq C \int_{\mathbb{R}^{2}}\left(p_{t-s}(x-(y+z))-p_{t-s}(x-y)\right)^{2}|z|^{2 H-2} d y d z \\
& \leq C(t-s)^{-1+H}
\end{aligned}
$$

which further implies, by the dominated convergence theorem, that

$$
\lim _{k \rightarrow \infty} \int_{0}^{t} D_{1}^{k}(t, s, x) d s=0 .
$$


Now let us look at $D_{2}^{k}(t, s, x)$. For fixed $y, z$, it holds that

$$
\left[\left(\sigma\left(u^{f_{n_{k}}}(s, y+z)\right)-\sigma(u(s, y+z))\right)-\left(\sigma\left(u^{f_{n_{k}}}(s, y)\right)-\sigma(u(s, y))\right)\right]^{2} \rightarrow 0
$$

as $k \rightarrow \infty$. In view of (4.58), (4.61) the following bound holds true:

$$
\begin{aligned}
& {\left[\left(\sigma\left(u^{f_{n_{k}}}(s, y+z)\right)-\sigma(u(s, y+z))\right)-\left(\sigma\left(u^{f_{n_{k}}}(s, y)\right)-\sigma(u(s, y))\right)\right]^{2}} \\
& \quad \leq C\left[\mid u^{\left.f_{n_{k}}(s, y+z)-\left.u^{f_{n_{k}}}(s, y)\right|^{2}+|u(s, y+z)-u(s, y)|^{2}\right]}\right. \\
& \quad \leq C\left(\left\|u^{f_{n_{k}}}(s, \cdot)\right\|_{\infty}^{2}+\|u(s, \cdot)\|_{\infty}^{2}\right) \chi_{\{|z| \geq 1\}}+C|z|^{2 H} \chi_{\{|z|<1\}} \\
& \quad \leq C\left\{\chi_{\{|z| \geq 1\}}+C|z|^{2 H} \chi_{\{|z|<1\}}\right\} .
\end{aligned}
$$

As $p_{t-s}^{2}(x-y)\left\{\chi_{\{|z| \geq 1\}}+C|z|^{2 H} \chi_{\{|z|<1\}}\right\}$ is integrable w.r.t. $|z|^{2 H-2} d y d z$, it follows from the dominated convergence theorem that

$$
\lim _{k \rightarrow \infty} D_{2}^{k}(t, s, x)=0
$$

Moreover,

$$
\begin{aligned}
D_{2}^{k}(t, s, x) & \leq C \int_{\mathbb{R}^{2}} p_{t-s}^{2}(x-y)\left\{\chi_{\{|z| \geq 1\}}+C|z|^{2 H} \chi_{\{|z|<1\}}\right\} d y d z \\
& \leq C(t-s)^{-\frac{1}{2}}
\end{aligned}
$$

Apply the dominated convergence theorem for the second time to get

$$
\lim _{k \rightarrow \infty} \int_{0}^{t} D_{2}^{k}(t, s, x) d s=0 .
$$

Combining (4.64), (4.68) and (4.73) together, we prove (4.63). Since $f_{n_{k}}$ converges weakly to $f$, using (4.63) we have

$$
\begin{aligned}
& \lim _{k \rightarrow \infty} \int_{0}^{t}\left\langle p_{t-s}(x-\cdot) \sigma\left(u^{f_{n_{k}}}(s, \cdot)\right), f_{n_{k}}(s, \cdot)\right\rangle_{\mathcal{H}} d s \\
&= \lim _{k \rightarrow \infty} \int_{0}^{t}\left\langle p_{t-s}(x-\cdot) \sigma\left(u^{f_{n_{k}}}(s, \cdot)\right)-p_{t-s}(x-\cdot) \sigma(u(s, \cdot)), f_{n_{k}}(s, \cdot)\right\rangle_{\mathcal{H}} d s \\
& \quad+\lim _{k \rightarrow \infty} \int_{0}^{t}\left\langle p_{t-s}(x-\cdot) \sigma(u(s, \cdot)), f_{n_{k}}(s, \cdot)\right\rangle_{\mathcal{H}} d s \\
&=\int_{0}^{t}\left\langle p_{t-s}(x-\cdot) \sigma(u(s, \cdot)), f(s, \cdot)\right\rangle_{\mathcal{H}} d s .
\end{aligned}
$$


Now let $k \rightarrow \infty$ in (4.62) to get

$$
u(t, x)=p_{t} u_{0}(x)+\int_{0}^{t}\left\langle p_{t-s}(x-\cdot) \sigma(u(s, \cdot)), f(s, \cdot)\right\rangle_{\mathcal{H}} d s,
$$

which implies $u=u^{f}$. To conclude that $u^{f_{n}} \rightarrow u^{f}$ in $X_{T}^{\frac{1}{2}-H}$, it suffices to show that the family $\left\{u^{f_{n}}, n \geq 1\right\}$ is relatively compact. According to Proposition 4.18 in [13], one only need to check the following three conditions (i) $\sup _{n}\left|u^{f_{n}}(0,0)\right|$ is finite. (ii) For every $x \in \mathbb{R},\left\{u^{f_{n}}(\cdot, x), n \geq 1\right\}$ is equicontinuous in time. (iii) For every $R>0$,

$$
\lim _{\delta \rightarrow 0} \sup _{n} \sup _{t \in[0, T], x \in[-R, R]} \int_{-\delta}^{\delta} \frac{\left|u^{f_{n}}(t, x+y)-u^{f_{n}}(t, x)\right|^{2}}{|y|^{2-2 H}} d y=0 .
$$

(i) Is clear. (ii) and (iii) follow easily from (4.58).

\section{Large deviation principle}

For $u \in X_{T}^{\frac{1}{2}-H}$, set

$$
S^{u}=\left\{f \in L^{2}([0, T] ; \mathcal{H}) ; u^{f}=u\right\},
$$

where $u^{f}$ stands for the solution to equation (4.1). Define

$$
I(u)=\inf _{f \in S^{u}}\left\{\frac{1}{2} \int_{0}^{T}\|f(s)\|_{\mathcal{H}}^{2} d s\right\} .
$$

Theorem 5.1. Assume that the assumptions of Theorem 2.7 hold. Then, the laws $\mu_{\varepsilon}$ of $\left\{u^{\varepsilon}(\cdot, \cdot), \varepsilon>0\right\}$ satisfy a large deviation principle on $X_{T}^{\frac{1}{2}-H}$ with the good rate function I $(\cdot)$. More precisely, we have

(i) For any closed subset $C \subset X_{T}^{\frac{1}{2}-H}$,

$$
\limsup _{\varepsilon \rightarrow 0} \varepsilon \log \mu_{\varepsilon}(C) \leq-\inf _{f \in C} I(f) .
$$

(ii) For any open set $G \subset X_{T}^{\frac{1}{2}-H}$,

$$
\liminf _{\varepsilon \rightarrow 0} \varepsilon \log \mu_{\varepsilon}(G) \geq-\inf _{f \in G} I(f) .
$$

Proof. By Theorem 2.7, there exists a unique strong (in the probabilistic sense) solution to the stochastic heat equation (1.1) in the space $X_{T}^{\frac{1}{2}-H}$. Therefore, for every $\varepsilon>0$, there exists a measurable mappings $\mathcal{G}^{\varepsilon}$ from $\mathbb{V}=C([0, T], \mathcal{H})$ to $\mathbb{U}:=X_{T}^{\frac{1}{2}-H}$ such that

$$
u^{\varepsilon}=\mathcal{G}^{\varepsilon}(W)
$$


where $W$ is the Gaussian process considered as an $\mathcal{H}$-valued cylindrical Brownian motion. Let $\mathcal{G}^{0}$ be defined as in Section 4 (just before Theorem 4.3). To prove the large deviation principle, we will verify the Assumption A in Section 3. The part (a) of the assumption is already proved in Theorem 4.3. Next, we will prove the part (b). Now fix $N>0$, and let $\psi_{\varepsilon}, \psi \in \mathcal{U}^{N}$ be such that $\psi_{\varepsilon}$ converges in distribution to $\psi$ as $\varepsilon \rightarrow 0$, where $\mathcal{U}^{N}$ is defined in Section 3. It is easy to see that $v^{\varepsilon}:=\mathcal{G}^{\varepsilon}\left(W+\frac{1}{\sqrt{\varepsilon}} \int_{0}^{\cdot} \psi_{\varepsilon}(s) d s\right)$ is the solution of the stochastic heat equation:

$$
\frac{\partial v^{\varepsilon}}{\partial t}=\frac{\kappa}{2} \frac{\partial^{2} v^{\varepsilon}}{\partial x^{2}}+\sqrt{\varepsilon} \sigma\left(v^{\varepsilon}\right) \dot{W}+\sigma\left(v^{\varepsilon}\right) \cdot \psi_{\varepsilon}, \quad t \geq 0, x \in \mathbb{R},
$$

equivalently in a mild form

$$
\begin{aligned}
v^{\varepsilon}(t, x)= & p_{t} u_{0}(x)+\sqrt{\varepsilon} \int_{0}^{t} \int_{\mathbb{R}} p_{t-s}(x-y) \sigma\left(v^{\varepsilon}(s, y)\right) W(d s, d y) \\
& +\int_{0}^{t}\left\langle p_{t-s}(x-\cdot) \sigma\left(v^{\varepsilon}(s, \cdot)\right), \psi_{\varepsilon}(s)\right\rangle_{\mathcal{H}} d s
\end{aligned}
$$

Our aim is to show that $v^{\varepsilon}$ converges in distribution to $v:=\mathcal{G}^{0}\left(\int_{0}^{\cdot} \psi(s) d s\right)$, the unique solution of the equation

$$
v(t, x)=p_{t} u_{o}(x)+\int_{0}^{t}\left\langle p_{t-s}(x-\cdot) \sigma(v(s, \cdot)), \psi(s)\right\rangle_{\mathcal{H}} d s .
$$

First, we claim that there exists $\theta>0$ such that

$$
\sup _{\varepsilon}\left\|v^{\varepsilon}\right\|_{\mathfrak{X}_{T, \theta}^{p}}<\infty
$$

where $\|\cdot\|_{\mathfrak{X}_{T, \theta}^{p}}$ is defined by (2.10). Let us now prove the claim. Write

$$
v^{\varepsilon}(t, x)=p_{t} u_{0}(x)+\Phi_{1}^{\varepsilon}(t, x)+\Phi_{2}^{\varepsilon}(t, x),
$$

where

$$
\begin{aligned}
& \Phi_{1}^{\varepsilon}(t, x):=\sqrt{\varepsilon} \int_{0}^{t} \int_{\mathbb{R}} p_{t-s}(x-y) \sigma\left(v^{\varepsilon}(s, y)\right) W(d s, d y), \\
& \Phi_{2}^{\varepsilon}(t, x):=\int_{0}^{t}\left\langle p_{t-s}(x-\cdot) \sigma\left(v^{\varepsilon}(s, \cdot)\right), \psi_{\varepsilon}(s)\right\rangle_{\mathcal{H}} d s .
\end{aligned}
$$

Applying Proposition 3.6 in [13] with $\beta=\frac{1}{2}-H$, it holds that

$$
\left\|\Phi_{1}^{\varepsilon}\right\|_{\mathfrak{X}_{T, \theta}^{p}} \leq C_{0} \sqrt{p}\left\|v^{\varepsilon}\right\|_{\mathfrak{X}_{T, \theta}^{p}}\left(\kappa^{\frac{H}{2}-\frac{1}{2}} \theta^{-\frac{H}{2}}+\kappa^{-\frac{1}{4}} \theta^{-\frac{1}{4}}+\kappa^{H-\frac{3}{4}} \theta^{\frac{1}{4}-H}\right),
$$


where $C_{0}$ is a constant depending only on $H$ and the Lipschitz constant of $\sigma$. We can get a similar bound for $\Phi_{2}$. Since $\psi_{\varepsilon} \in \mathcal{U}^{N}$ we have

$$
\begin{aligned}
\left|\Phi_{2}^{\varepsilon}(t, x)\right| \leq & \left(\int_{0}^{t}\left\|p_{t-s}(x-\cdot) \sigma\left(v^{\varepsilon}(s, \cdot)\right)\right\|_{\mathcal{H}}^{2} d s\right)^{\frac{1}{2}}\left(\int_{0}^{t}\left\|\psi_{\varepsilon}(s)\right\|_{\mathcal{H}}^{2} d s\right)^{\frac{1}{2}} \\
\leq & c_{H} N^{\frac{1}{2}}\left(\int_{0}^{t} \int_{\mathbb{R}^{2}} \mid p_{t-s}(x-y-z) \sigma\left(v^{\varepsilon}(s, y+z)\right)\right. \\
& \left.-\left.p_{t-s}(x-y) \sigma\left(v^{\varepsilon}(s, y)\right)\right|^{2}|z|^{2 H-2} d y d z d s\right)^{\frac{1}{2}} \\
\leq & C N^{\frac{1}{2}}\left(\int_{0}^{t} \int_{\mathbb{R}^{2}}\left|p_{t-s}(x-y-z)-p_{t-s}(x-y)\right|^{2}\right. \\
& \left.\times\left|v^{\varepsilon}(s, y+z)\right|^{2}|z|^{2 H-2} d y d z\right)^{\frac{1}{2}} \\
+ & C N^{\frac{1}{2}}\left(\int_{0}^{t} \int_{\mathbb{R}^{2}} p_{t-s}^{2}(x-y)\left|v^{\varepsilon}(s, y+z)-v^{\varepsilon}(s, y)\right|^{2}|z|^{2 H-2} d y d z d s\right)^{\frac{1}{2}} .
\end{aligned}
$$

By Minkovski inequality it follows from (5.8) that

$$
\begin{aligned}
& \left\|\Phi_{2}^{\varepsilon}(t, x)\right\|_{L^{p}(\Omega)} \\
& \leq C N^{\frac{1}{2}}\left(\int_{0}^{t} \int_{\mathbb{R}^{2}}\left|p_{t-s}(x-y-z)-p_{t-s}(x-y)\right|^{2}\right. \\
& \left.\quad \times\left\|v^{\varepsilon}(s, y+z)\right\|_{L^{p}(\Omega)}^{2}|z|^{2 H-2} d y d z\right)^{\frac{1}{2}} \\
& \quad+C N^{\frac{1}{2}}\left(\int_{0}^{t} \int_{\mathbb{R}^{2}} p_{t-s}^{2}(x-y)\left\|v^{\varepsilon}(s, y+z)-v^{\varepsilon}(s, y)\right\|_{L^{p}(\Omega)}^{2}|z|^{2 H-2} d y d z d s\right)^{\frac{1}{2}} .
\end{aligned}
$$

Similar estimates can be obtained for

$$
\left\|\Phi_{2}^{\varepsilon}(t, x+h)-\Phi_{2}^{\varepsilon}(t, x)\right\|_{L^{p}(\Omega)},
$$

which are needed to control the norm in the space $\mathfrak{X}_{T, \theta}^{p}$. We have

$$
\begin{aligned}
& \left\|\Phi_{2}^{\varepsilon}(t, x+h)-\Phi_{2}^{\varepsilon}(t, x)\right\|_{L^{p}(\Omega)} \\
& \leq C\left(\int_{0}^{t} \int_{\mathbb{R}^{2}} \mid\left(p_{t-s}(x+h-y-z)-p_{t-s}(x-y-z)\right)\right. \\
& \left.\quad-\left.\left(p_{t-s}(x+h-y)-p_{t-s}(x-y)\right)\right|^{2}\left\|v^{\varepsilon}(s, y+z)\right\|_{L^{p}(\Omega)}^{2}|z|^{2 H-2} d y d z\right)^{\frac{1}{2}}
\end{aligned}
$$




$$
\begin{aligned}
& +C\left(\int_{0}^{t} \int_{\mathbb{R}^{2}}\left|p_{t-s}(x+h-y)-p_{t-s}(x-y)\right|^{2}\right. \\
& \left.\times\left\|v^{\varepsilon}(s, y+z)-v^{\varepsilon}(s, y)\right\|_{L^{p}(\Omega)}^{2}|z|^{2 H-2} d y d z d s\right)^{\frac{1}{2}} .
\end{aligned}
$$

Due to the above estimates (5.9), (5.10), we can now follow exactly the same proof as Proposition 3.6 in [13] to obtain

$$
\left\|\Phi_{2}^{\varepsilon}\right\|_{\mathfrak{X}_{T, \theta}^{p}} \leq C_{1}\left\|v^{\varepsilon}\right\|_{\mathfrak{X}_{T, \theta}^{p}}\left(k^{\frac{H}{2}-\frac{1}{2}} \theta^{-\frac{H}{2}}+k^{-\frac{1}{4}} \theta^{-\frac{1}{4}}+k^{H-\frac{3}{4}} \theta^{\frac{1}{4}-H}\right),
$$

where $C_{1}$ is a constant depending only on $H, N$ and the Lipschitz constant of $\sigma$. Now combining (5.7), (5.11) and (5.6) we get

$$
\begin{aligned}
\left\|v^{\varepsilon}\right\|_{\mathfrak{X}_{T, \theta}^{p}} \leq & C+C_{1}\left\|v^{\varepsilon}\right\|_{\mathfrak{X}_{T, \theta}^{p}}\left(k^{\frac{H}{2}-\frac{1}{2}} \theta^{-\frac{H}{2}}+k^{-\frac{1}{4}} \theta^{-\frac{1}{4}}+k^{H-\frac{3}{4}} \theta^{\frac{1}{4}-H}\right) \\
& +C_{0} \sqrt{p}\left\|v^{\varepsilon}\right\|_{\mathfrak{X}_{T, \theta}^{p}}\left(k^{\frac{H}{2}-\frac{1}{2}} \theta^{-\frac{H}{2}}+k^{-\frac{1}{4}} \theta^{-\frac{1}{4}}+k^{H-\frac{3}{4}} \theta^{\frac{1}{4}-H}\right) .
\end{aligned}
$$

Now choosing $\theta>0$ sufficiently large so that

$$
\left(C_{0} \sqrt{p}+C_{1}\right)\left(k^{\frac{H}{2}-\frac{1}{2}} \theta^{-\frac{H}{2}}+k^{-\frac{1}{4}} \theta^{-\frac{1}{4}}+k^{H-\frac{3}{4}} \theta^{\frac{1}{4}-H}\right)<1,
$$

it follows from (5.12) that

$$
\sup _{\varepsilon}\left\|v^{\varepsilon}\right\|_{\mathfrak{X}_{T, \theta}^{p}}<\infty
$$

which is the claim.

To prove our theorem, we need a stronger conclusion. For any $\beta<H$ and $p \geq 2$, it holds that

$$
\sup _{\varepsilon}\left\|v^{\varepsilon}\right\|_{\mathfrak{X}_{T, \theta}^{\beta, p}}<\infty
$$

where

$$
\|f\|_{\mathfrak{X}_{T, \theta}^{\beta, p}}:=\sup _{t \in[0, T], x \in \mathbb{R}} e^{-\theta t}\left\|v^{\varepsilon}(t, x)\right\|_{L^{p}(\Omega)}+\sup _{t \in[0, T], x \in \mathbb{R}} e^{-\theta t} \mathcal{N}_{\beta}^{L^{p}(\Omega)} v^{\varepsilon}(t, x) .
$$

In fact, let $\Phi_{1}^{\varepsilon}, \Phi_{2}^{\varepsilon}$ be defined as above. By Proposition 3.6 in [13], we have, for large enough $\theta$,

$$
\sup _{\varepsilon}\left\|\Phi_{i}^{\varepsilon}\right\|_{\mathfrak{X}_{T, \theta}^{\beta, p}} \leq C_{i}\left\|\sigma\left(v^{\varepsilon}\right)\right\|_{\mathfrak{X}_{T, \theta}^{p}} \leq C\left\|v^{\varepsilon}\right\|_{\mathfrak{X}_{T, \theta}^{p}}
$$

Then (5.13) follows from (5.5), (5.6) and (5.14).

Applying Proposition 3.8 in [13] to $\Phi_{1}^{\varepsilon}, \Phi_{2}^{\varepsilon}$ we get that

$$
\begin{aligned}
\left\|v^{\varepsilon}\left(t_{2}, x_{2}\right)-v^{\varepsilon}\left(t_{1}, x_{1}\right)\right\|_{L^{p}(\Omega)} & \leq C\left\|\sigma\left(v^{\varepsilon}\right)\right\|_{\mathfrak{X}_{T, \theta}^{p}}\left\{\left|t_{2}-t_{1}\right|^{\frac{H}{2}}+\left|x_{2}-x_{1}\right|^{H}\right\} \\
& \leq C\left\|v^{\varepsilon}\right\|_{\mathfrak{X}_{T, \theta}^{p}}\left\{\left|t_{2}-t_{1}\right|^{\frac{H}{2}}+\left|x_{2}-x_{1}\right|^{H}\right\} \\
& \leq C\left\{\left|t_{2}-t_{1}\right|^{\frac{H}{2}}+\left|x_{2}-x_{1}\right|^{H}\right\},
\end{aligned}
$$


where (5.5) was used and $C$ is an independent constant. By (5.13), (5.15) and Proposition 4.24 in [13] we conclude that the laws of the family $\left\{v^{\varepsilon}, \varepsilon>0\right\}$ is tight on the space $X_{T}^{\frac{1}{2}-H}$. Hence, the family $\left\{\left(v^{\varepsilon}, W(\cdot, \cdot), \psi_{\varepsilon}\right), \varepsilon>0\right\}$ is tight on the space

$$
X_{T}^{\frac{1}{2}-H} \times C([0, T] \times \mathbb{R}) \times L^{2}([0, T] ; \mathcal{H}) .
$$

Recall that the topology of weak convergence is used for $L^{2}([0, T] ; \mathcal{H})$. Choosing a subsequence if necessary, by Skorokhod's embedding theorem, there exists a probability space $(\bar{\Omega}, \overline{\mathcal{F}}, \bar{P})$ carrying a family of random fields $\left(\bar{v}^{\varepsilon}, \bar{W}^{\varepsilon}(\cdot, \cdot), \bar{\psi}_{\varepsilon}\right)$ such that

$$
\left(\bar{v}^{\varepsilon}, \bar{W}^{\varepsilon}, \bar{\psi}_{\varepsilon}\right)=\left(v^{\varepsilon}, W, \psi_{\varepsilon}\right)
$$

in law and $\bar{P}$-almost surely, $\left(\bar{v}^{\varepsilon}, \bar{W}^{\varepsilon}(\cdot, \cdot), \bar{\psi}^{\varepsilon}\right)$ converges to some random fields $(\bar{v}, \bar{W}(\cdot, \cdot), \bar{\psi})$ in the space

$$
X_{T}^{\frac{1}{2}-H} \times C([0, T] \times \mathbb{R}) \times L^{2}([0, T] ; \mathcal{H}) .
$$

In particular, the following stochastic heat equation is held for $\left(\bar{v}^{\varepsilon}, \bar{\psi}^{\varepsilon}, \bar{W}^{\varepsilon}\right)$ :

$$
\begin{aligned}
\bar{v}^{\varepsilon}(t, x)= & p_{t} u_{0}(x) \\
& +\sqrt{\varepsilon} \int_{0}^{t} \int_{\mathbb{R}} p_{t-s}(x-y) \sigma\left(\bar{v}^{\varepsilon}(s, y)\right) \bar{W}^{\varepsilon}(d s, d y) \\
& +\int_{0}^{t}\left\langle p_{t-s}(x-\cdot) \sigma\left(\bar{v}^{\varepsilon}(s, \cdot)\right), \bar{\psi}_{\varepsilon}(s)\right\rangle_{\mathcal{H}} d s .
\end{aligned}
$$

Next we want to pass to the limit in (5.16) as $\varepsilon \rightarrow 0$. First of all, we have

$$
\begin{aligned}
\bar{E} & {\left[\left|\sqrt{\varepsilon} \int_{0}^{t} \int_{\mathbb{R}} p_{t-s}(x-y) \sigma\left(\bar{v}^{\varepsilon}(s, y)\right) \bar{W}^{\varepsilon}(d s, d y)\right|^{2}\right] } \\
& =\varepsilon E\left[\int_{0}^{t}\left\|p_{t-s}(x-\cdot) \sigma\left(\bar{v}^{\varepsilon}(s, \cdot)\right)\right\|_{\mathcal{H}}^{2} d s\right],
\end{aligned}
$$

where $\bar{E}$ stands for the expectation under the probability measure $\bar{P}$. By the Lipschitz continuity of $\sigma$ it is easy to see that

$$
\begin{aligned}
& \left\|p_{t-s}(x-\cdot) \sigma\left(\bar{v}^{\varepsilon}(s, \cdot)\right)\right\|_{\mathcal{H}}^{2} \\
& \leq C \int_{\mathbb{R}^{2}}\left(p_{t-s}(x-(y+z))-p_{t-s}(x-y)\right)^{2}\left|\bar{v}^{\varepsilon}(s, y+z)\right|^{2}|z|^{2 H-2} d y d z \\
& \quad+C \int_{\mathbb{R}^{2}} p_{t-s}^{2}(x-y)\left|\bar{v}^{\varepsilon}(s, y+z)-\bar{v}^{\varepsilon}(s, y)\right|^{2}|z|^{2 H-2} d y d z
\end{aligned}
$$


Thus, we have

$$
\begin{aligned}
\bar{E}[\mid & \left.\left.\sqrt{\varepsilon} \int_{0}^{t} \int_{\mathbb{R}} p_{t-s}(x-y) \sigma\left(\bar{v}^{\varepsilon}(s, y)\right) \bar{W}^{\varepsilon}(d s, d y)\right|^{2}\right] \\
\leq & C \varepsilon \int_{0}^{t} \int_{\mathbb{R}^{2}}\left(p_{t-s}(x-(y+z))-p_{t-s}(x-y)\right)^{2}\left\|\bar{v}^{\varepsilon}(s, y+z)\right\|_{L^{2}(\Omega)}^{2}|z|^{2 H-2} d y d z \\
& +C \varepsilon \int_{0}^{t} \int_{\mathbb{R}^{2}} p_{t-s}^{2}(x-y)\left\|\bar{v}^{\varepsilon}(s, y+z)-\bar{v}^{\varepsilon}(s, y)\right\|_{L^{2}(\Omega)}^{2}|z|^{2 H-2} d y d z \\
\leq & C \varepsilon \int_{0}^{t} d s \int_{\mathbb{R}^{2}}\left(p_{t-s}(x-(y+z))-p_{t-s}(x-y)\right)^{2}\left\|\bar{v}^{\varepsilon}\right\|_{\mathfrak{X}_{T, \theta}^{2}}^{2}|z|^{2 H-2} d y d z \\
& +C \varepsilon \int_{0}^{t} d s \int_{\mathbb{R}^{2}} p_{t-s}^{2}(x-y)\left\|\bar{v}^{\varepsilon}\right\|_{\mathfrak{X}_{T, \theta}^{2}}^{2} d y \\
\leq & C \varepsilon \int_{0}^{t}\left\{(t-s)^{-1+H}+(t-s)^{-\frac{1}{2}}\right\} d s \rightarrow 0
\end{aligned}
$$

as $\varepsilon \rightarrow 0$, where we have used Lemma 3.1 and the fact $\sup _{\varepsilon}\left\|\bar{v}^{\varepsilon}\right\|_{\mathfrak{X}_{T, \theta}^{2}}^{2}<\infty$. Next, we will prove

$$
\lim _{\varepsilon \rightarrow 0} \int_{0}^{t}\left\langle p_{t-s}(x-\cdot) \sigma\left(\bar{v}^{\varepsilon}(s, \cdot)\right), \bar{\psi}_{\varepsilon}(s)\right\rangle_{\mathcal{H}} d s=\int_{0}^{t}\left\langle p_{t-s}(x-\cdot) \sigma(\bar{v}(s, \cdot)), \bar{\psi}(s)\right\rangle_{\mathcal{H}} d s .
$$

Since $\bar{\psi}_{\varepsilon} \rightarrow \bar{\psi}$ weakly in $L^{2}([0, T]: \mathcal{H})$ and since $\int_{0}^{T}\left\|\bar{\psi}_{\varepsilon}\right\|_{\mathcal{H}}^{2} d s \leq N$, to prove (5.20) it suffices to show

$$
\lim _{\varepsilon \rightarrow 0} \int_{0}^{t} \bar{E}\left[\left\|p_{t-s}(x-\cdot)\left[\sigma\left(\bar{v}^{\varepsilon}(s, \cdot)\right)-\sigma(\bar{v}(s, \cdot))\right]\right\|_{\mathcal{H}}^{2} d s\right]=0
$$

Now,

$$
\begin{aligned}
\bar{E}\left[\left\|p_{t-s}(x-\cdot)\left(\sigma\left(\bar{v}^{\varepsilon}(s, \cdot)\right)-\sigma(\bar{v}(s, \cdot))\right)\right\|_{\mathcal{H}}^{2}\right] \\
\leq C \int_{\mathbb{R}^{2}}\left(p_{t-s}(x-(y+z))-p_{t-s}(x-y)\right)^{2} \\
\quad \times \bar{E}\left[\left(\sigma\left(\bar{v}^{\varepsilon}(s, y+z)\right)-\sigma(\bar{v}(s, y+z))\right)^{2}\right]|z|^{2 H-2} d y d z \\
\quad+C \int_{\mathbb{R}^{2}} p_{t-s}^{2}(x-y) \bar{E}\left\{\left[\left(\sigma\left(\bar{v}^{\varepsilon}(s, y+z)\right)-\sigma(\bar{v}(s, y+z))\right)\right.\right. \\
\left.\left.\quad-\left(\sigma\left(\bar{v}^{\varepsilon}(s, y)\right)-\sigma(\bar{v}(s, y))\right)\right]^{2}\right\}|z|^{2 H-2} d y d z \\
:=G_{1}^{k}(t, s, x)+G_{2}^{k}(t, s, x) .
\end{aligned}
$$


For every $y, z$, clearly $\left(\sigma\left(\bar{v}^{\varepsilon}(s, y+z)\right)-\sigma(\bar{v}(s, y+z))\right)^{2} \rightarrow 0$ as $k \rightarrow \infty$. On the other hand, for $p>2$,

$$
\sup _{\varepsilon} \bar{E}\left[\left|\sigma\left(\bar{v}^{\varepsilon}(s, y+z)\right)-\sigma(\bar{v}(s, y+z))\right|^{p}\right] \leq C\left[\sup _{\varepsilon}\left\|\bar{v}^{\varepsilon}\right\|_{\mathfrak{X}_{T, \theta}^{p}}^{p}+\|\bar{v}\|_{\mathfrak{X}_{T, \theta}^{p}}^{p}\right]<\infty
$$

Hence, we have $\bar{E}\left[\left(\sigma\left(\bar{v}^{\varepsilon}(s, y+z)\right)-\sigma(\bar{v}(s, y+z))\right)^{2}\right] \rightarrow 0$. Note that

$$
\begin{aligned}
& \left(p_{t-s}(x-(y+z))-p_{t-s}(x-y)\right)^{2} \bar{E}\left[\left(\sigma\left(\bar{v}^{\varepsilon}(s, y+z)\right)-\sigma(\bar{v}(s, y+z))\right)^{2}\right]|z|^{2 H-2} \\
& \quad \leq C\left[\sup _{\varepsilon}\left\|\bar{v}^{\varepsilon}\right\|_{\mathfrak{X}_{T, \theta}^{2}}^{2}+\|\bar{v}\|_{\mathfrak{X}_{T, \theta}^{2}}^{2}\right]\left(p_{t-s}(x-(y+z))-p_{t-s}(x-y)\right)^{2}|z|^{2 H-2} \\
& \quad \leq C\left(p_{t-s}(x-(y+z))-p_{t-s}(x-y)\right)^{2}|z|^{2 H-2},
\end{aligned}
$$

where the right-hand side is $d y d z$-integrable. Applying the dominated convergence theorem, we deduce that

$$
\lim _{k \rightarrow \infty} G_{1}^{k}(t, s, x)=0
$$

Furthermore, by Lemma 3.1 we have

$$
\begin{aligned}
G_{1}^{k}(t, s, x) & \leq C \int_{\mathbb{R}^{2}}\left(p_{t-s}(x-(y+z))-p_{t-s}(x-y)\right)^{2}|z|^{2 H-2} d y d z \\
& \leq C(t-s)^{-1+H}
\end{aligned}
$$

which further implies, by the dominated convergence theorem, that

$$
\lim _{k \rightarrow \infty} \int_{0}^{t} G_{1}^{k}(t, s, x) d s=0 .
$$

Now let us look at $G_{2}^{k}(t, s, x)$. For fixed $y, z$, similarly we have

$$
\bar{E}\left[\left(\sigma\left(\bar{v}^{\varepsilon}(s, y+z)\right)-\sigma(\bar{v}(s, y+z))\right)-\left(\sigma\left(\bar{v}^{\varepsilon}(s, y)\right)-\sigma(\bar{v}(s, y))\right)\right]^{2} \rightarrow 0
$$

as $k \rightarrow \infty$. In view of (5.5), (5.15) we also have

$$
\begin{aligned}
& \bar{E}\left[\left[\left(\sigma\left(\bar{v}^{\varepsilon}(s, y+z)\right)-\sigma(\bar{v}(s, y+z))\right)-\left(\sigma\left(\bar{v}^{\varepsilon}(s, y)\right)-\sigma\left(\bar{v}^{\varepsilon}(s, y)\right)\right)\right]^{2}\right] \\
& \leq C\left[\bar{E}\left[\left|\bar{v}^{\varepsilon}(s, y+z)-\bar{v}^{\varepsilon}(s, y)\right|^{2}\right]+\bar{E}\left[|\bar{v}(s, y+z)-\bar{v}(s, y)|^{2}\right]\right] \\
& \quad \leq C\left(\left[\sup _{\varepsilon}\left\|\bar{v}^{\varepsilon}\right\|_{\mathfrak{X}_{T, \theta}^{2}}^{2}+\|\bar{v}\|_{\mathfrak{X}_{T, \theta}^{2}}^{2}\right]\right) \chi_{\{|z| \geq 1\}}+C|z|^{2 H} \chi_{\{|z|<1\}} \\
& \quad \leq C\left\{\chi_{\{|z| \geq 1\}}+C|z|^{2 H} \chi_{\{|z|<1\}}\right\} .
\end{aligned}
$$

As $p_{t-s}^{2}(x-y)\left\{\chi_{\{|z| \geq 1\}}+C|z|^{2 H} \chi_{\{|z|<1\}}\right\}$ is integrable w.r.t. $|z|^{2 H-2} d y d z$, it follows from the dominated convergence theorem that

$$
\lim _{k \rightarrow \infty} G_{2}^{k}(t, s, x)=0
$$


Moreover,

$$
\begin{aligned}
G_{2}^{k}(t, s, x) & \leq C \int_{\mathbb{R}^{2}} p_{t-s}^{2}(x-y)\left\{\chi_{\{|z| \geq 1\}}+C|z|^{2 H} \chi_{\{|z|<1\}}\right\}|z|^{2 H-2} d y d z \\
& \leq C(t-s)^{-\frac{1}{2}}
\end{aligned}
$$

Apply the dominated convergence theorem the second time to get

$$
\lim _{k \rightarrow \infty} \int_{0}^{t} G_{2}^{k}(t, s, x) d s=0
$$

(5.31) and (5.26) yield (5.21), and hence (5.20). Now let $\varepsilon \rightarrow 0$ in (5.16) and use (5.19), (5.20) to conclude that

$$
\bar{v}(t, x)=p_{t} u_{0}(x)+\int_{0}^{t}\left\langle p_{t-s}(x-\cdot) \sigma(\bar{v}(s, \cdot)), \bar{\psi}(s)\right\rangle_{\mathcal{H}} d s .
$$

Since $\psi^{\varepsilon} \rightarrow \psi$ in distribution and $\bar{\psi}^{\varepsilon}$ has the same law as $\psi^{\varepsilon}, \bar{\psi}$ must have the same law as $\psi$. It follows from the uniqueness of the solution of equation $(5.32)$ that $v(\cdot, \cdot)$, the solution of the equation (5.4), and $\bar{v}(\cdot, \cdot)$ will have the same law. We finally can conclude

$$
v^{\varepsilon} \rightarrow v
$$

in distribution, completing the proof of the theorem.

\section{Acknowledgements}

We thank the referee for the very careful reading and useful comments. This work is partially supported by a grant from the Simons Foundation \#209206, the NSF Grant DMS1512891, the ARO Grant FED0070445 and NSFC Grant (No. 11431014).

\section{References}

[1] Balan, R.M., Jolis, M. and Quer-Sardanyons, L.S. (2015). SPDEs with affine multiplicative fractional noise in space with index $\frac{1}{4}<H<\frac{1}{2}$. Electron. J. Probab. 2036 . MR3354614

[2] Budhiraja, A. and Dupuis, P. (2000). A variational representation for positive functionals of infinite dimensional Brownian motion. Probab. Math. Statist. 20 139-161.

[3] Budhiraja, A., Dupuis, P. and Maroulas, V. (2008). Large deviations for infinite dimensional stochastic dynamical systems. Ann. Probab. 36 1390-1420. MR2435853

[4] Chenal, F. and Millet, A. (1997). Uniform large deviations for parabolic SPDEs and applications. Stochastic Process. Appl. 72 161-186. MR1486551

[5] Chueshov, I. and Millet, A. (2010). Stochastic 2D hydrodynamical type systems: Well posedness and large deviations. Appl. Math. Optim. 61 379-420. MR2609596

[6] Dalang, R. and Quer-Sardanyons, L. (2011). Stochastic integrals for spde's: A comparison. Expo. Math. 29 67-109. 
[7] Dalang, R.C. (1999). Extending the martingale measure stochastic integral with applications to spatially homogeneous s.p.d.e.'s. Electron. J. Probab. 4 29. MR1684157

[8] Dalang, R.C. (2001). Corrections to: "Extending the martingale measure stochastic integral with applications to spatially homogeneous s.p.d.e.'s" [Electron J. Probab. 4 (1999), no. 6, 29 pp. (electronic); MR1684157 (2000b:60132)]. Electron. J. Probab. 6 5. MR1825714

[9] Da Prato, G. and Zabczyk, J. (1992). Stochastic Equations in Infinite Dimensions. Cambridge: Cambridge Univ. Press.

[10] Dupuis, P. and Ellis, R.S. (1997). A Weak Convergence Approach to the Theory of Large Deviations. New York: Wiley. MR1431744

[11] Gyöngy, I. (1998). Existence and uniqueness results for semilinear stochastic partial differential equations. Stochastic Process. Appl. 73 271-299. MR1608641

[12] Gyöngy, I. and Nualart, D. (1999). On the stochastic Burgers' equation in the real line. Ann. Probab. 27 782-802. MR1698967

[13] Hu, Y., Huang, J., Lê, K., Nualart, D. and Tindel, S. (2015). Stochastic heat equation with rough dependence in space. Preprint.

[14] Peszat, S. and Zabczyk, J. (1997). Stochastic evolution equations with a spatially homogeneous Wiener process. Stochastic Process. Appl. 72 187-204. MR1486552

[15] Pipiras, V. and Taqqu, M.S. (2000). Integration questions related to fractional Brownian motion. Probab. Theory Related Fields 118 251-291. MR1790083

[16] Sritharan, S.S. and Sundar, P. (2006). Large deviations for the two-dimensional Navier-Stokes equations with multiplicative noise. Stochastic Process. Appl. 116 1636-1659.

[17] Xu, T. and Zhang, T. (2009). White noise driven SPDEs with reflection: Existence, uniqueness and large deviation principles. Stochastic Process. Appl. 119 3453-3470.

Received September 2015 and revised June 2016 